\title{
The potential impact of enhanced accident tolerant cladding materials on reactivity initiated
}

\section{accidents in light water reactors}

Nicholas R. Brown ${ }^{1, *}$, Aaron J. Wysocki ${ }^{1}$, Kurt A. Terrani ${ }^{1}$, Kevin G. Xu ${ }^{1, \ddagger}$, Daniel M. Wachs ${ }^{2}$

${ }^{1}$ Oak Ridge National Laboratory, 1 Bethel Valley Rd, Oak Ridge, TN, 37831, USA;

${ }^{2}$ Idaho National Laboratory, P.O. Box 1625, Idaho Falls, ID 83415-6188, USA;

*Corresponding author: Nicholas R. Brown, brownnr@ornl.gov

"Student Intern from the University of Michigan

\begin{abstract}
Advanced cladding materials with potentially enhanced accident tolerance will yield different light-water reactor performance and safety characteristics than the present zirconium-based cladding alloys. These differences are due to cladding material properties, reactor physics, and thermal hydraulics characteristics. Differences in reactor physics are driven by the fundamental properties (e.g., neutron absorption cross section in iron for an iron-based cladding) and also by design modifications necessitated by the candidate cladding materials (e.g., a larger fuel pellet to compensate for parasitic absorption).

This paper describes three-dimensional nodal kinetics simulations of a reactivity-initiated accident (RIA) in a representative pressurized water reactor with both nuclear-grade iron-chromium-aluminum (FeCrAl) and silicon-carbide fiber silicon carbide ceramic matrix composite ( $\mathrm{SiC} / \mathrm{SiC})$ materials. This study shows similar RIA neutronic behavior for $\mathrm{SiC} / \mathrm{SiC}$ cladding configurations versus reference Zircaloy cladding. However, the FeCrAl cladding response indicates similar energy deposition but with significantly shorter pulses of higher magnitude. This is

This manuscript has been authored by UT-Battelle, LLC under Contract No. DE-AC05-00OR22725 with the U.S. Department of Energy. The United States Government retains and the publisher, by accepting the article for publication, acknowledges that the United States Government retains a non-exclusive, paid-up, irrevocable, world-wide license to publish or reproduce the published form of this manuscript, or allow others to do so, for United States Government purposes. The Department of Energy will provide public access to these results of federally sponsored research in accordance with the DOE Public Access Plan (http://energy.gov/downloads/doe-public-access-plan ).
\end{abstract}


due to the shorter neutron generation time of the core models based on FeCrAl cladding. The FeCrAl-based cases exhibit a more rapid fuel thermal expansion rate than other cases, and the resultant pellet-cladding interaction may occur more rapidly. This study shows that utilizing different cladding material and fuel design make a difference for RIA response, including core response and fuel response.

Key Words: transient testing, nodal kinetics, reactivity initiated accident, fuel thermal expansion, accident tolerant cladding.

\section{Introduction}

The reactivity-initiated accident (RIA) is a postulated design basis accident (DBA) in a light water reactor (LWR). The RIA takes the form of a control-rod-ejection accident in a pressurized water reactors (PWRs) or a control rod drop accident in a boiling water reactors (BWRs). The US Nuclear Regulatory Commission (NRC) General Design Criteria require that postulated RIAs must not damage the reactor coolant pressure boundary, and they must not significantly impair core coolability (Electric Power Research Institute [EPRI] 2002) as outlined in 10 CFR Part 50: Domestic licensing of

production and utilization facilities. One possible cladding failure mode during RIA is pellet cladding mechanical interaction (PCMI), which occurs on a very rapid (millisecond) timescale

Since the events at Fukushima Daiichi in 2011, the US Department of Energy Office of Nuclear Energy (DOE-NE) Advanced Fuels Campaign (AFC) has been studying nuclear fuel and cladding materials with potentially enhanced accident tolerance (Goldner 2012, Terrani et al. 2014, Zinkle et al. 2014). These fuel and cladding materials will affect the progression of RIA response. The focus of this paper is on the expected impact of two advanced cladding candidates, FeCrAl alloy and $\mathrm{SiC} / \mathrm{SiC}$ 
composite, on the progression of a control-rod-ejection accident in a PWR at hot zero power (HZP) conditions.

Understanding the impact of an RIA is very important for candidate fuel/cladding concepts with new materials. RIA proceeds in two phases (Desquines et al. 2012). The first phase is also called the low-temperature phase, and the cladding performance is dominated by PCMI. The first phase occurs rapidly as the fuel pellet thermally expands due to the intense quasi-adiabatic energy deposition of the overpower transient. This is caused by a pulse in the fission rate in the fuel, which is turned around by the Doppler effect. Thus, the mechanical response of the cladding is mostly driven by the dimensional change of the fuel pellet during an RIA transient. The extent and rate of dimensional change in the fuel pellet is proportional to the energy deposited during the RIA. The mechanical response of the fuel pin therefore depends strongly on the reactor kinetics.

The second phase is the high-temperature phase that coincides with departure from nucleate boiling and the subsequent film boiling for a PWR. In this regime, heat is transferred from the pellet, through the cladding, and into the coolant. Because of the large amount of energy deposited in the fuel, a boiling crisis during transport to the coolant is possible. If a boiling crisis occurs, a vapor film forms on the cladding surface, which impedes heat transfer to the coolant and drives up the fuel temperature. Fuel can fail due to melting or due to brittle fracture during the quench phase.

The safety criteria formulated originally in the 1970s based mostly on US data focused only on this second phase since the early tests conducted on fresh or low burnup fuel segments did not reveal the potential for failure due to PCMI (U.S. Atomic Energy Commission 1974). Accordingly, a limit of $170 \mathrm{cal} / \mathrm{g}$ of energy deposition in the oxide fuel was prescribed. However, international tests 
conducted during and after 1990s on fuel segments with medium to high burnup indicated the susceptibility to fuel failure at lower energy deposition thresholds and post-test examinations indicated that failure mechanism was due to PCMI (Alvis 2010). This was the case since at these burnup levels the gap between the fuel and cladding had decreased greatly or disappeared altogether while the ductility of the cladding had also plummeted due to radiation damage as well as hydrogen pickup (Fuketa 2012, Sugiyama 2009). Accordingly, In the US an amendment to acceptance criteria defined in NUREG-0800 was made (U.S. Nuclear Regulatory Commission 2007) to ensure compliance with General Design Criterion (GDC) 10, within Appendix A to 10 CFR Part 50 along with similar modifications made internationally.

Both phases of an RIA could cause cladding failure. The focus of this paper is on PCMI that is the responsible mechanism for failure during the first phase. Some of the differences in RIA response in an LWR with an advanced cladding will be driven by the fundamental properties (e.g., neutron absorption cross section in iron for an iron-based cladding) and others by design modifications necessitated by the candidate cladding materials (e.g., a larger fuel pellet to compensate for parasitic absorption).

This paper describes an effort to use reactor core kinetics simulation capabilities to estimate differences in the ATF concept pulse response characteristics during hypothetical RIA events. The results of this study apply to future in-pile transient testing as well as to out-of-pile separate effects tests. Essentially these results will be used to inform future experimental studies with appropriate test conditions to study mechanical response of the cladding.

The applicable conditions have been determined using three-dimensional nodal kinetics 
simulations of an RIA in a representative PWR with both $\mathrm{FeCrAl}$ and SiC/SiC cladding materials. The study yields pulse shape boundary conditions for use in future mechanical tests of candidate materials to simulate the RIA event, specifically peak unconstrained fuel thermal expansion and unconstrained fuel thermal expansion rate during the power pulse following the rod ejection. Other example information of interest includes the pulse width and the energy deposition in the fuel. The effort spans the parameter space of potential $\mathrm{FeCrAl}$ and $\mathrm{SiC} / \mathrm{SiC}$ cladding design concepts for PWRs (Brown et al. 2015, George et al. 2015, Todosow et al. 2015; Younker and Fratoni 2016).

\section{Core Modeling Approach}

This work informs on the qualitative and quantitative differences in RIA response of two leading candidate-cladding materials versus the reference zirconium-based cladding. The parameters that describe the conditions an RIA imposes on fuel and cladding include energy deposition, pulse magnitude, pulse width, and rate of fuel temperature increase. Not only do different cladding materials and fuel designs lead to different responses to these parameters, but they also influence the values of the parameters themselves (i.e., core loads of different fuel types responding differently to rod insertion or rod drop). These quantities, then, inform on differences relative to zirconium-based cladding in the boundary conditions of a PCMI separate effects test. The intent of the PCMI tests is to determine the failure strain limit $(\%)$ for a particular cladding. The pulse width and rate of fuel temperature increase help determine the thermal expansion rates (\%/second) experienced by the fuel pellets which can be used to estimate the displacement and displacement rate in the cladding for a variety of fuel concepts. 
To generate these parameters, a three-dimensional PWR core model was developed for several candidate configurations: a reference Zircaloy-4 cladding case, two FeCrAl cladding configurations with parameters from the literature, and two $\mathrm{SiC} / \mathrm{SiC}$ cladding configurations with parameters from the literature. In this context the differences between the configurations were the cladding material, fuel pellet diameter, and cladding thickness. The details of a similar methodology are outlined in Brown et al. (2014) in which different lattice configurations are used, along with a set of cross section branch parameters (fuel temperature, moderator density, moderator temperature, soluble boron concentration, and control rod) that bound hot zero power conditions. For each of the candidate configurations, an equilibrium cycle core model was generated using the Purdue Advanced Reactor Core Simulator (PARCS) (Downar et al. 2002), the US NRC's reactor-core simulator. PARCS is a well-established tool for simulation of RIA. Example applications of PARCS for RIA include Kozlowski and Downar (2006), as well as Hursin et al. (2013).

The equilibrium core configurations were calculated with a single burnable poison configuration in a multicycle calculation. An identical integral fuel burnable absorber (IFBA) configuration was used for all fuel assemblies. Within the fuel assemblies, 112 of the fuel pins used IFBA coating for reactivity control. The SCALE 6.1 lattice physics tool TRITON/NEWT was used for the generation of two-group cross sections (DeHart and Bowman 2011). TRITON/NEWT was used with ENDF/B-VII.0 238-group cross sections collapsed to a problem-specific 49-group library. The selected branch parameters include conditions required to accurately simulate both hot full power and HZP conditions. The $17 \times 17$ Westinghouse assembly geometry used in the generation of the few-group cross sections for the PARCS model is shown in Figure 1. 


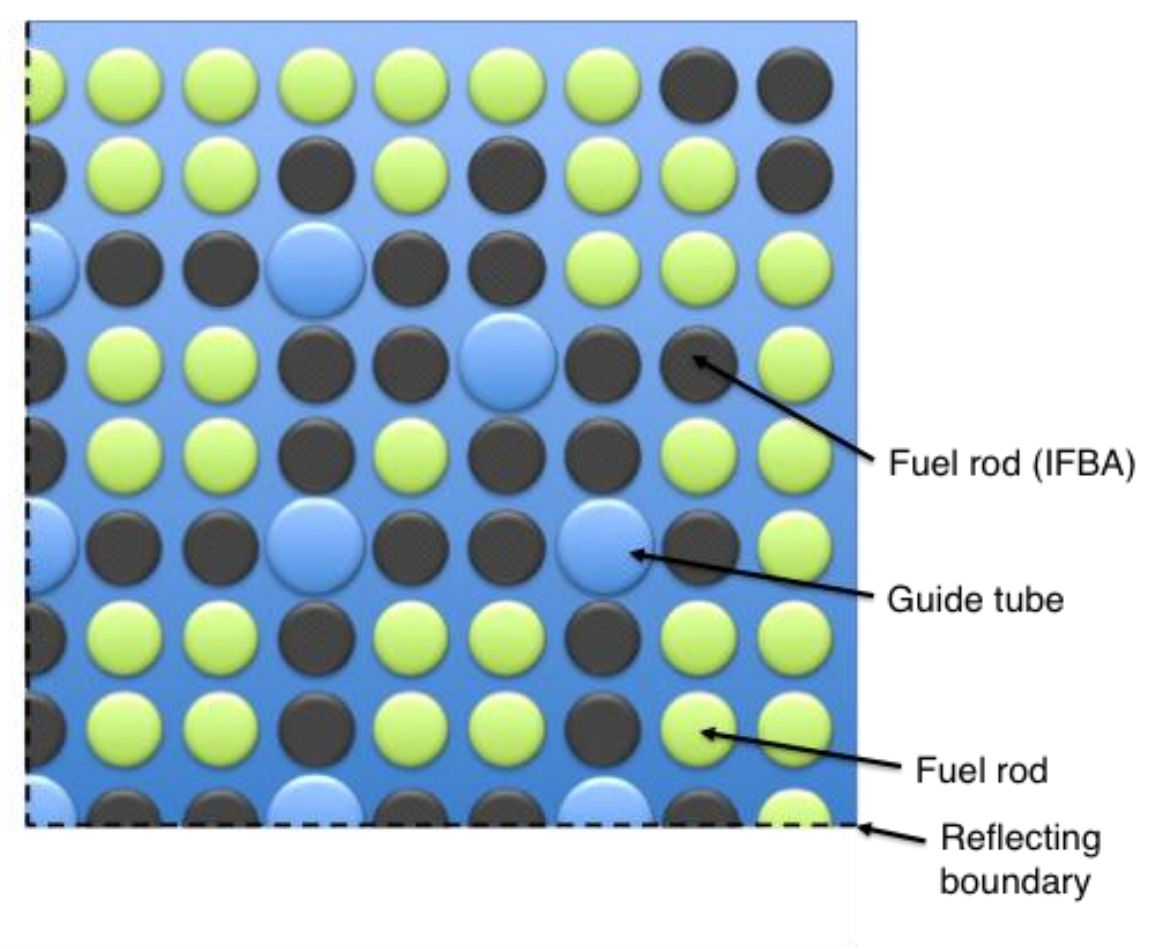

Figure 1. One-quarter-assembly geometry used for generation of the few-group parameters for the reactor core models (Brown et al. 2014).

The lattice configurations considered as part of this study are shown in Table 1. The parameters are based on those used for scoping calculations in Brown et al. (2014), Brown et al. (2015), George et al. (2015), and Todosow et al. (2015). Differences from the reference Zircaloy cladding case are displayed in bold. The composition of the $\mathrm{FeCrAl}$ cladding assumes $71 \%$ iron, 21\% chromium, 5\% aluminum, and 3\% molybdenum. Due to its relatively high chromium and molybdenum content, this FeCrAl composition is expected to bound the neutronic impacts of FeCrAl cladding. It should be noted that the chromium content of an eventual nuclear-grade FeCrAl would likely be lower (Yamamoto et al. 2015), which will reduce parasitic neutron absorption. Results from detailed 
calculations of the parasitic neutron absorption in FeCrAl are presented in Brown et al. (2015) and George et al. (2015).

Table 1. Fuel lattice configurations considered in generation of core models (differences versus reference case are bolded).

\begin{tabular}{lccccc}
\hline Cladding material & Zircaloy & SiC/SiC & SiC/SiC & FeCrAl & FeCrAl \\
\hline Fuel pellet radius (mm) & 4.096 & 4.096 & $\mathbf{3 . 9 0 6}$ & $\mathbf{4 . 2 4 9}$ & $\mathbf{4 . 3 6 8}$ \\
Cladding outer radius (mm) & 4.750 & 4.750 & 4.750 & 4.750 & 4.750 \\
Cladding thickness (mm) & 0.572 & 0.572 & $\mathbf{0 . 7 6 2}$ & $\mathbf{0 . 4 1 9}$ & $\mathbf{0 . 3 0 0}$ \\
Gap thickness (mm) & 0.082 & 0.082 & 0.082 & 0.082 & 0.082 \\
Enrichment $(\%)$ & $5.0 \%$ & $5.0 \%$ & $5.0 \%$ & $\mathbf{5 . 5 \%}$ & $5.0 \%$ \\
\hline
\end{tabular}

The PARCS multicycle capability was used to calculate the equilibrium core models with a discharge burnup convergence criterion of $0.1 \mathrm{GWd} / \mathrm{t}$. There were 157 assemblies in the core model, with a total thermal power of $3400 \mathrm{MW}$. In each cycle, the relevant fuel assemblies were shuffled according to a prescribed three-batch fuel-management scheme targeting an 18-month cycle length (Brown et al. 2014). Only one fuel type was used in this simplified core model; with an actual core design, multiple fuel types with varying ${ }^{235} \mathrm{U}$ enrichments and burnable poison content would be used in a reactor core. Soluble boron was used for reactivity control throughout the cycle, and control rods were not used throughout the cycle. The critical soluble boron concentration was calculated iteratively within each burnup step. As the fuel depleted, the core simulator calculated the boron 
letdown curve for the core configuration. The equilibrium core search was performed with few-group cross sections that included branch conditions for fuel temperature, moderator density and temperature, and soluble boron feedback. For each core calculation, the relevant thermal properties (volumetric heat capacity and thermal conductivity) of the cladding materials were used in the core simulator (Brown et al. 2015). For SiC/SiC cladding, an expression for the saturated irradiated thermal conductivity was used that was derived from available experimental data (Katoh et al. 2014). The characteristics of the reactor core considered in these scoping calculations are shown in Table 2.

Table 2. Parameters used in the core analyses (Brown et al. 2014).

\begin{tabular}{lc}
\hline \multicolumn{1}{c}{ Parameter } & Value \\
\hline Reactor core heat output, MW(th) & 3,400 \\
\hline System pressure, nominal, MPa & 15.51 \\
\hline Nominal inlet coolant temperature, ${ }^{\mathbf{0}} \mathbf{C}$ & 279.4 \\
\hline Fuel assembly design & $17 \times 17$ \\
\hline Active fuel height, cm & 426.7 \\
\hline Number of fuel assemblies & 157 \\
\hline Fuel assembly pitch, cm & 21.44 \\
\hline Uranium rods per assembly & 264 \\
\hline Number of control clusters & $53-$ rod cluster control assembly (RCCA) \\
\hline Control rod material & Ag-In-Cd \\
\hline Number of fuel batches & 3 \\
\hline
\end{tabular}




\section{Core Modeling Steady State Results}

The equilibrium core analyses yield detailed information about the reactor core throughout the cycle, including power peaking factors, critical boron concentration, reactivity coefficients, and kinetics parameters. For both $\mathrm{FeCrAl}$ and $\mathrm{SiC} / \mathrm{SiC}$ cladding materials, some reactor physics and reactor core analyses have been performed in the literature (Andrews et al. 2016, Brown et al. 2015, Carpenter 2010, Dobisesky 2011, George et al. 2015, Lee et al. 2013, Todosow et al. 2015). The objective of the present core analyses is to develop a consistent basis of comparison to understand the impact of these different cladding materials and associated lattice designs on RIA.

The critical boron concentration throughout the equilibrium cycle is shown in Figure 2. The radial assembly power peaking factor throughout the equilibrium cycle is shown in Figure 3 , and the axial power peaking factor is shown in Figure 4. The reactor core average specific burn-up in gigawatt days per initial heavy metal metric ton $(\mathrm{GWd} / \mathrm{t})$ is shown in Figure 5.

$10 / 55$ 


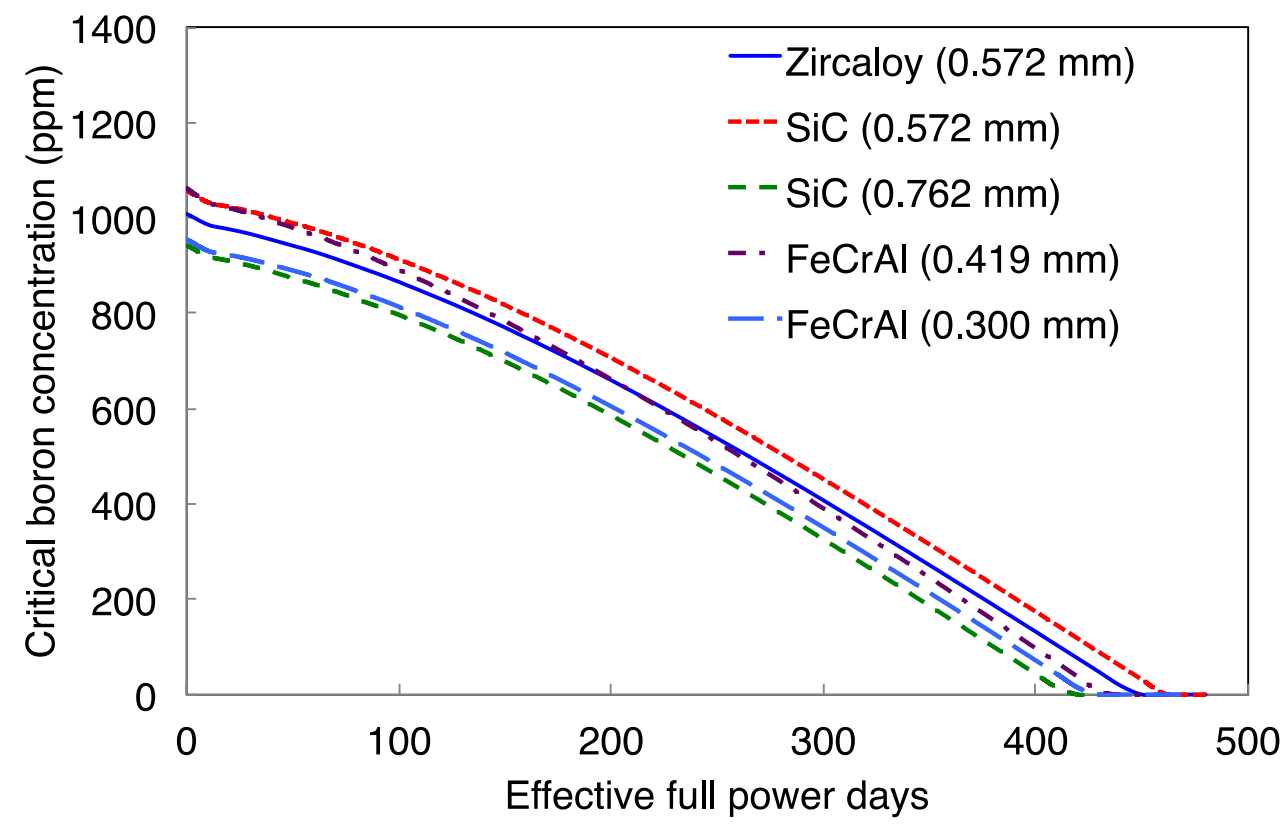

Figure 2. Critical boron concentration during the equilibrium cycle. 


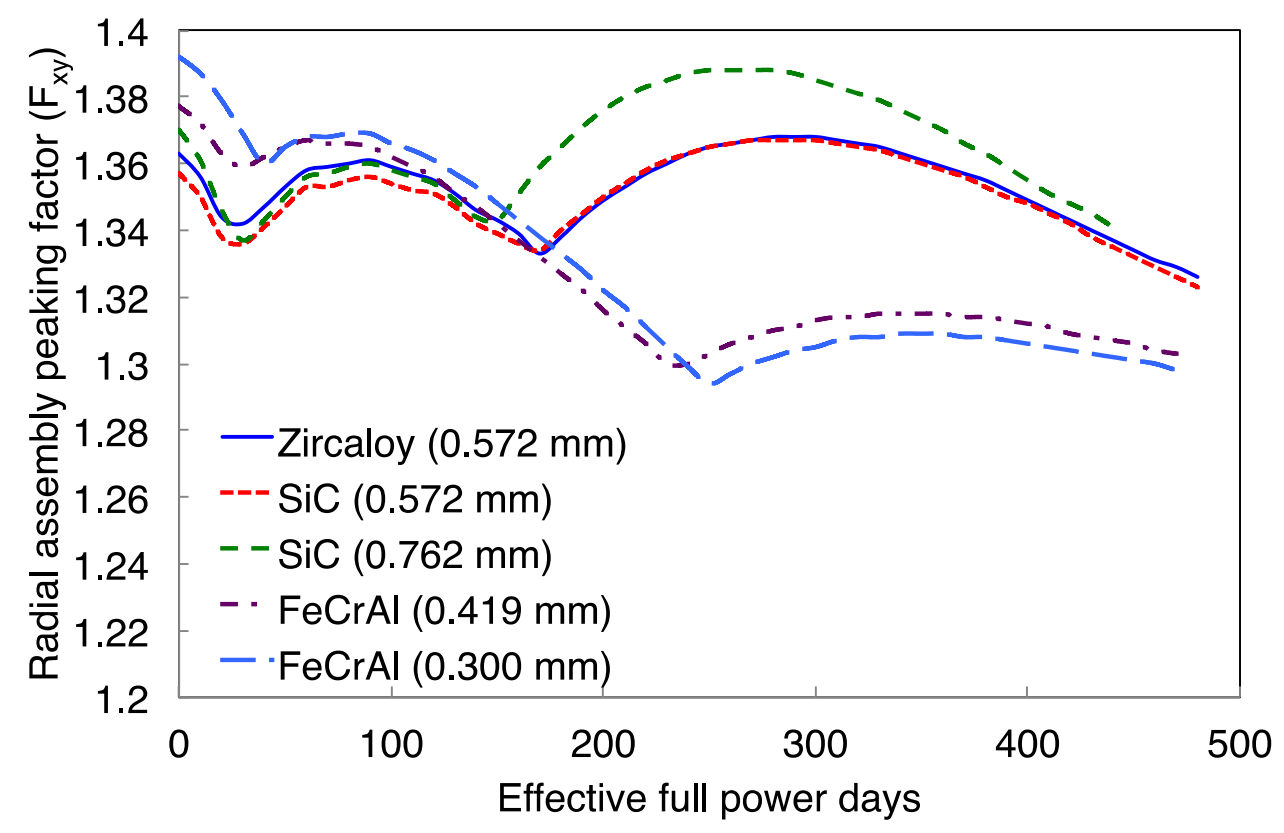

Figure 3. Radial assembly power peaking factor during the equilibrium cycle. 


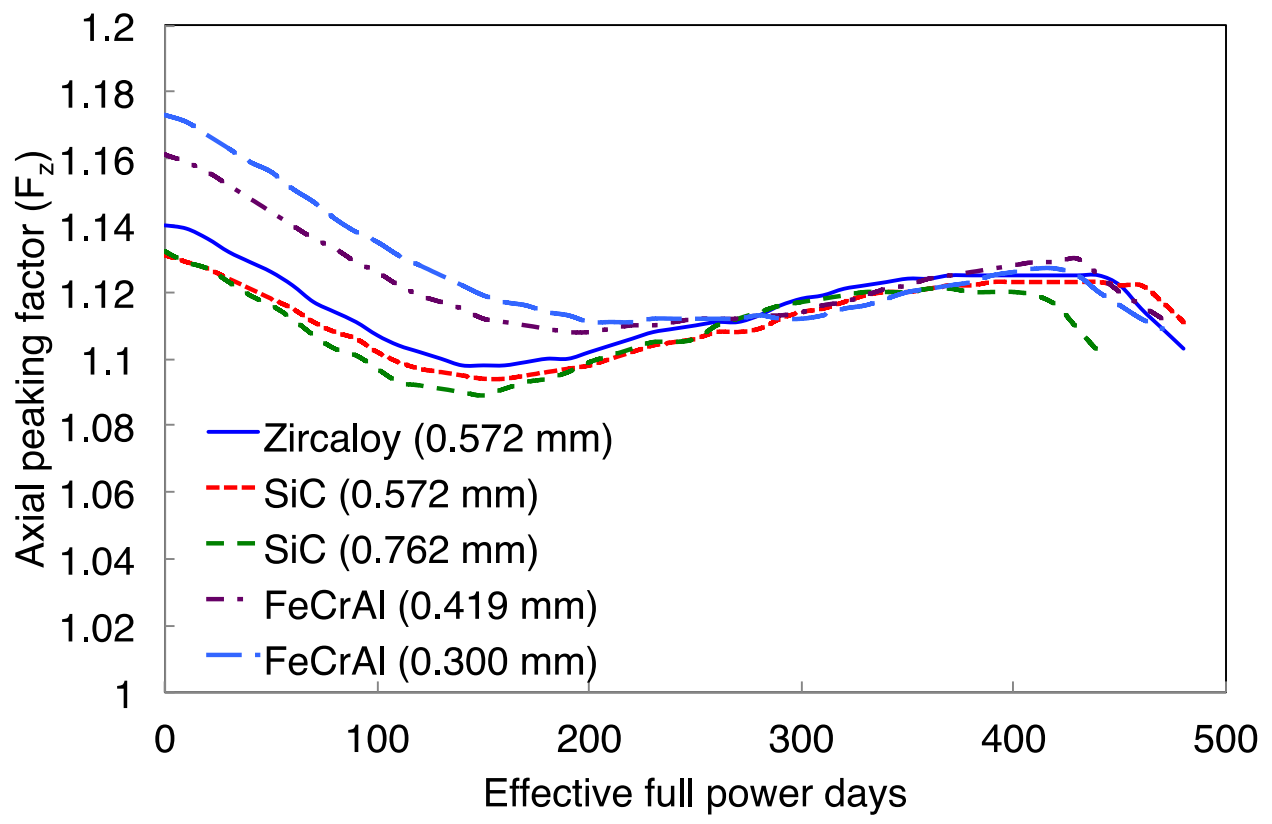

Figure 4. Axial power peaking factor during the equilibrium cycle. 


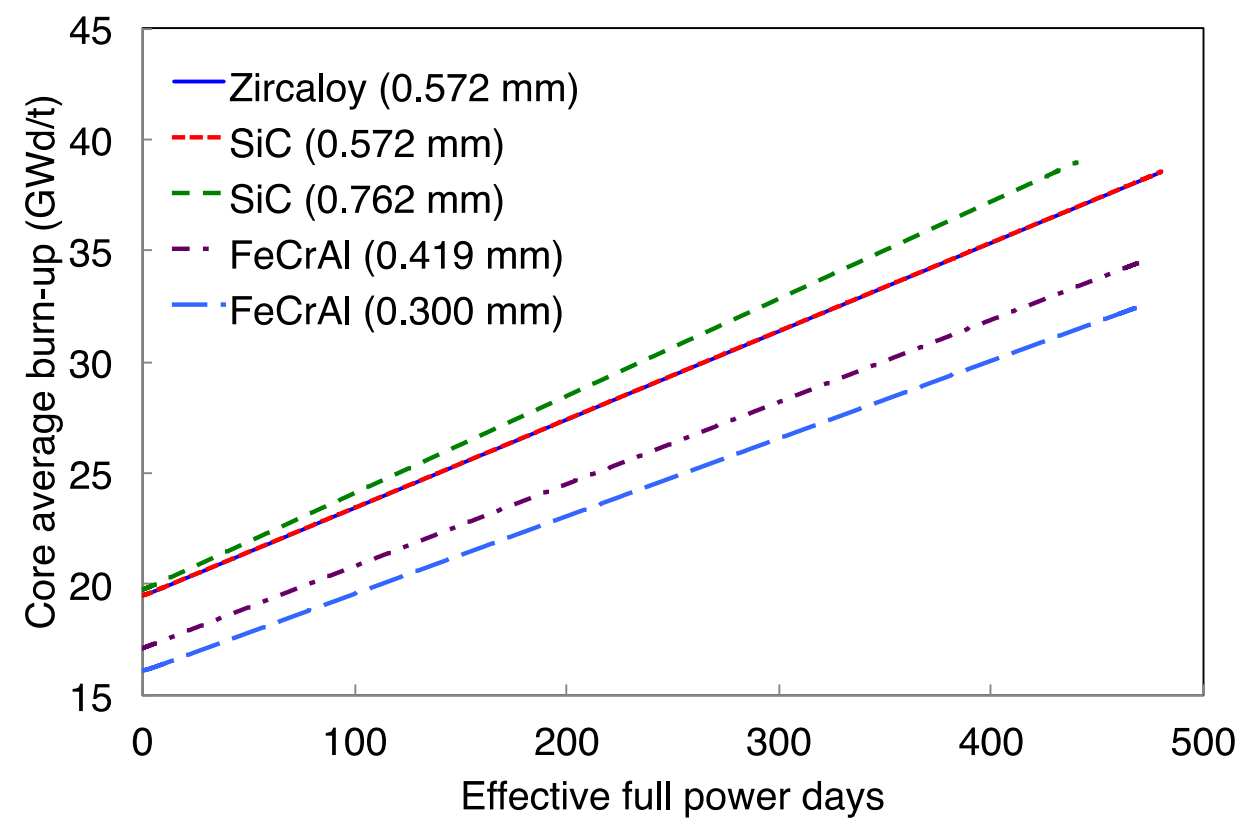

Figure 5. Core average specific burn-up $(\mathrm{GWd} / \mathrm{t})$ during the equilibrium cycle. Note: Zircaloy and SiC/SiC results overlap at the $0.572 \mathrm{~mm}$ cladding thickness.

The axial power distribution at beginning-of-cycle (BOC) is shown in Figure 6. This shows the impact of the different fuel-to-moderator ratio in the FeCrAl cases, which yields marginally stronger moderator density and temperature feedback effects and drives the power peak towards the bottom of the reactor core. This is consistent with the limited set of results presented in Brown et al. (2015). The axial power distribution at end-of-cycle (EOC) is shown in Figure 7. 


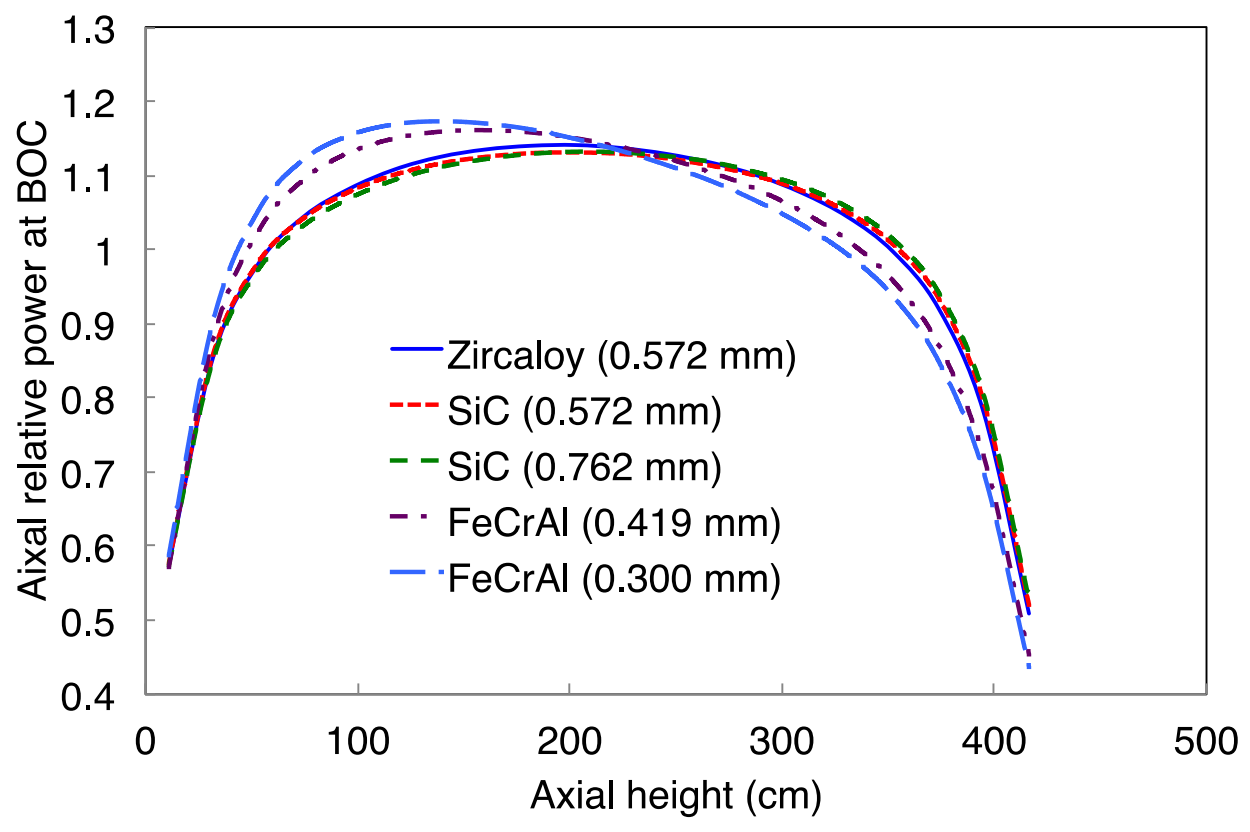

Figure 6. Axial relative power distribution at BOC. 


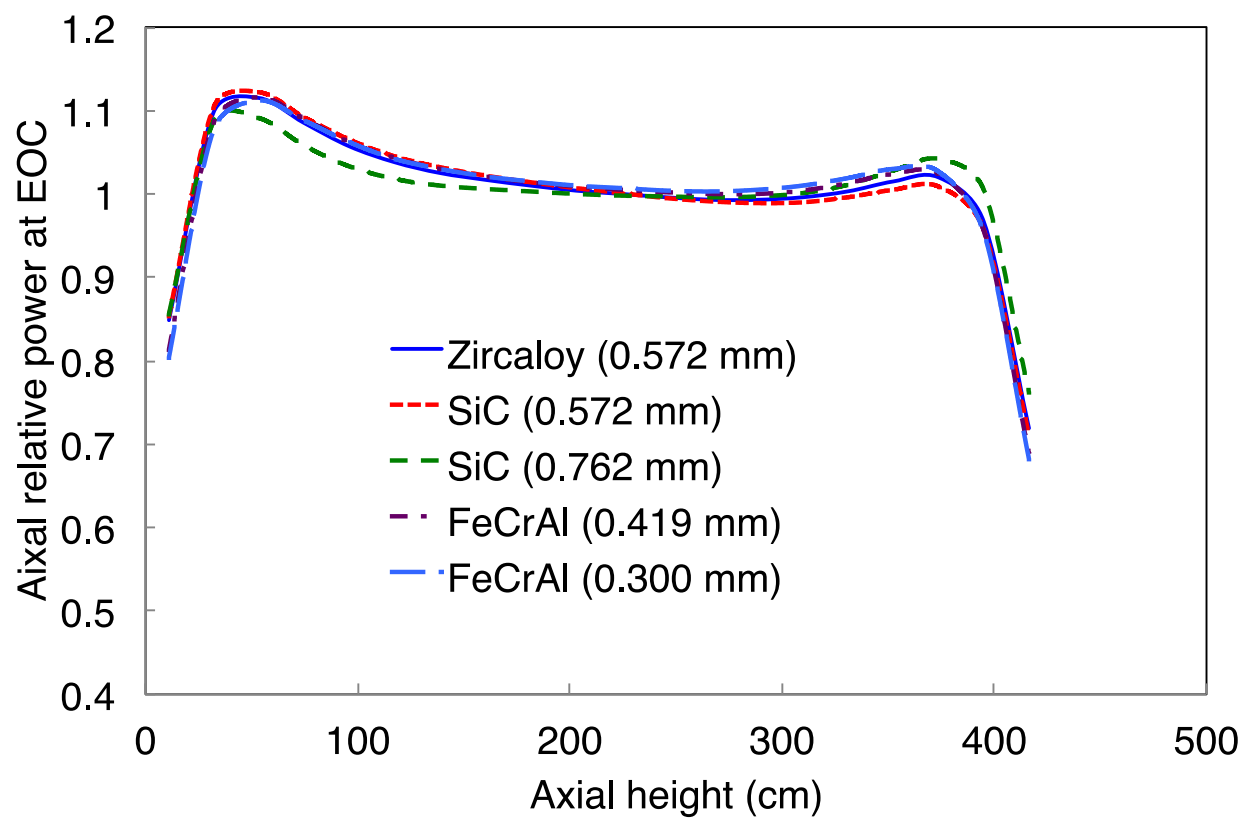

Figure 7. Axial relative power distribution at EOC.

The radial core power and burn-up distributions are shown at BOC and EOC for each core configuration in Figure 8-Figure 12. These power distributions show general similarity between each of the cases. 


\begin{tabular}{|c|c|c|c|c|c|}
\hline 0.97 & & & & \multirow{2}{*}{\multicolumn{2}{|c|}{ BOC 1/8-Core $\mathrm{UO}_{2}$}} \\
\hline 43.9 & & & & & \\
\hline 1.15 & 1.00 & & & \multicolumn{2}{|c|}{ Zircaloy $(0.572 \mathrm{~mm})$} \\
\hline 25.0 & 43.9 & & & 1.36 & Relative power \\
\hline 1.04 & 1.31 & 1.14 & & 19.8 & Burn-up (GWd/t) \\
\hline 45.6 & 0.0 & 41.7 & & & \\
\hline 1.29 & 1.36 & 1.34 & 1.24 & & \\
\hline 25.0 & 19.8 & 24.7 & 35.1 & & \\
\hline 1.04 & 0.98 & 1.13 & 1.34 & 1.14 & \\
\hline 35.7 & 45.3 & 36.3 & 14.5 & 15.6 & \\
\hline 0.88 & 1.12 & 1.17 & 0.84 & 0.65 & \\
\hline 41.7 & 0.0 & 13.2 & 36.6 & 0.0 & \\
\hline 0.78 & 0.92 & 0.76 & 0.51 & & \\
\hline 35.1 & 13.7 & 0.0 & 0.0 & & \\
\hline 0.51 & 0.45 & & & & \\
\hline 0.0 & 0.0 & & & & \\
\hline
\end{tabular}

\begin{tabular}{|c|c|c|c|c|c|}
\hline 0.75 & & & & \multirow{2}{*}{\multicolumn{2}{|c|}{ EOC $1 / 8$-Core $\mathrm{UO}_{2}$}} \\
\hline 58.8 & & & & & \\
\hline 0.91 & 0.83 & & & \multicolumn{2}{|c|}{ Zircaloy $(0.572 \mathrm{~mm})$} \\
\hline 43.2 & 60.4 & & & 1.33 & Relative power \\
\hline 0.85 & 1.21 & 0.89 & & 23.9 & Burn-up (GWd/t) \\
\hline 62.4 & 23.7 & 59.6 & & & \\
\hline 0.97 & 1.02 & 0.96 & 0.90 & & \\
\hline 44.8 & 40.9 & 44.6 & 53.5 & & \\
\hline 0.88 & 0.84 & 0.91 & 1.08 & 1.04 & \\
\hline 52.8 & 61.7 & 54.3 & 35.8 & 34.9 & \\
\hline 0.94 & 1.33 & 1.19 & 0.87 & 0.87 & \\
\hline 58.7 & 23.9 & 35.3 & 52.0 & 13.8 & \\
\hline 1.01 & 1.18 & 1.18 & 0.84 & & \\
\hline 52.2 & 34.1 & 18.9 & 12.6 & & \\
\hline 1.04 & 0.91 & & & & \\
\hline 14.8 & 13.0 & & & & \\
\hline
\end{tabular}

Figure 8. Radial power and burn-up distribution for $\mathrm{UO}_{2}$ fuel with Zircaloy-4 $(0.572 \mathrm{~mm})$ cladding at BOC (left) and EOC (right). 


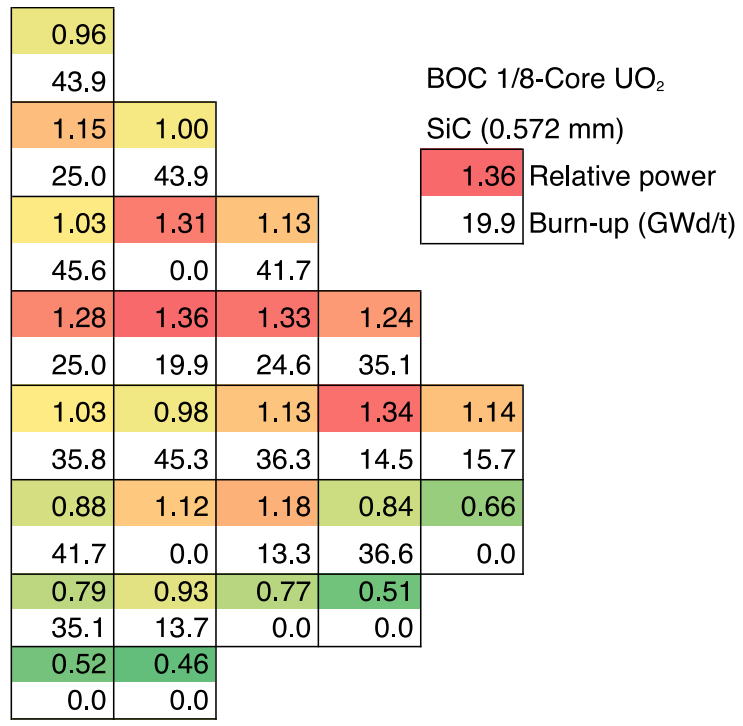

\begin{tabular}{|c|c|c|c|c|c|}
\hline 0.76 & & & & \multirow{3}{*}{\multicolumn{2}{|c|}{$\begin{array}{l}\text { EOC } 1 / 8-\text {-Core } \mathrm{UO}_{2} \\
\mathrm{SiC}(0.572 \mathrm{~mm})\end{array}$}} \\
\hline 58.8 & & & & & \\
\hline 0.91 & 0.84 & & & & \\
\hline 43.1 & 60.3 & & & 1.33 & Relative power \\
\hline 0.85 & 1.22 & 0.89 & & 24.0 & Burn-up (GWd/t) \\
\hline 62.4 & 23.7 & 59.5 & & & \\
\hline 0.97 & 1.02 & 0.97 & 0.90 & & \\
\hline 44.8 & 40.9 & 44.5 & 53.5 & & \\
\hline 0.88 & 0.85 & 0.91 & 1.08 & 1.04 & \\
\hline 52.9 & 61.6 & 54.3 & 35.8 & 34.9 & \\
\hline 0.93 & 1.33 & 1.19 & 0.87 & 0.87 & \\
\hline 58.7 & 24.0 & 35.4 & 52.1 & 13.8 & \\
\hline 1.00 & 1.18 & 1.17 & 0.84 & & \\
\hline 52.3 & 34.2 & 19.0 & 12.6 & & \\
\hline 1.04 & 0.91 & & & & \\
\hline 14.9 & 13.0 & & & & \\
\hline
\end{tabular}

Figure 9. Radial power and burn-up distribution for $\mathrm{UO} 2$ fuel with $\mathrm{SiC} / \mathrm{SiC}(0.572 \mathrm{~mm})$ cladding at BOC (left) and EOC (right). 


\begin{tabular}{|c|c|c|c|c|c|}
\hline 0.95 & & & & \multirow{2}{*}{\multicolumn{2}{|c|}{ BOC 1/8-Core $\mathrm{UO}_{2}$}} \\
\hline 44.3 & & & & & \\
\hline 1.14 & 0.98 & & & \multicolumn{2}{|c|}{$\mathrm{SiC}(0.762 \mathrm{~mm})$} \\
\hline 25.5 & 44.3 & & & 1.37 & Relative power \\
\hline 1.01 & 1.30 & 1.12 & & \multirow[t]{4}{*}{20.2} & Burn-up (GWd/t) \\
\hline 46.1 & 0.0 & 42.2 & & & \\
\hline 1.29 & 1.37 & 1.35 & 1.25 & & \\
\hline 25.5 & 20.2 & 24.9 & 35.7 & & \\
\hline 1.03 & 0.97 & 1.13 & 1.37 & 1.16 & \\
\hline 36.3 & 45.7 & 36.9 & 14.7 & 15.9 & \\
\hline 0.86 & 1.12 & 1.19 & 0.84 & 0.66 & \\
\hline 42.2 & 0.0 & 13.4 & 37.2 & 0.0 & \\
\hline 0.78 & 0.93 & 0.76 & 0.50 & & \\
\hline 35.7 & 13.9 & 0.0 & 0.0 & & \\
\hline 0.51 & 0.45 & & & & \\
\hline 0.0 & 0.0 & & & & \\
\hline
\end{tabular}

\begin{tabular}{|c|c|c|c|c|c|}
\hline 0.73 & & & & \multirow{3}{*}{\multicolumn{2}{|c|}{$\begin{array}{l}\text { EOC } 1 / 8 \text {-Core } \mathrm{UO}_{2} \\
\mathrm{SiC}(0.762 \mathrm{~mm})\end{array}$}} \\
\hline 60.2 & & & & & \\
\hline 0.89 & 0.81 & & & & \\
\hline 45.1 & 61.9 & & & 1.33 & Relative power \\
\hline 0.82 & 1.21 & 0.86 & & 26.6 & Burn-up (GWd/t) \\
\hline 64.0 & 26.0 & 61.4 & & & \\
\hline 0.95 & 1.00 & 0.95 & 0.88 & & \\
\hline 46.9 & 43.1 & 46.6 & 55.6 & & \\
\hline 0.86 & 0.82 & 0.89 & 1.08 & 1.04 & \\
\hline 54.8 & 63.3 & 56.4 & 38.1 & 37.2 & \\
\hline 0.92 & 1.33 & 1.19 & 0.87 & 0.90 & \\
\hline 60.7 & 26.6 & 37.9 & 54.1 & 15.5 & \\
\hline 1.00 & 1.19 & 1.20 & 0.87 & & \\
\hline 54.6 & 36.7 & 21.3 & 14.2 & & \\
\hline 1.08 & 0.95 & & & & \\
\hline 16.9 & 14.7 & & & & \\
\hline
\end{tabular}

Figure 10. Radial power and burn-up distribution for $\mathrm{UO}_{2}$ fuel with $\mathrm{SiC} / \mathrm{SiC}(0.762 \mathrm{~mm})$ cladding at BOC (left) and EOC (right). 


\begin{tabular}{|c|c|c|c|c|c|}
\hline 1.05 & & & & \multirow{2}{*}{\multicolumn{2}{|c|}{$\mathrm{BOC} 1 / 8$-Core $\mathrm{UO}_{2}$}} \\
\hline 40.0 & & & & & \\
\hline 1.21 & 1.08 & & & \multicolumn{2}{|c|}{$\mathrm{FeCrAl}(0.419 \mathrm{~mm})$} \\
\hline 21.9 & 40.0 & & & 1.38 & \multirow{13}{*}{$\begin{array}{l}\text { Relative power } \\
\text { Burn-up }(\mathrm{GWd} / \mathrm{t})\end{array}$} \\
\hline 1.11 & 1.34 & 1.20 & & 16.6 & \\
\hline 41.6 & 0.0 & 37.2 & & & \\
\hline 1.32 & 1.38 & 1.34 & 1.27 & & \\
\hline 21.9 & 16.6 & 22.5 & 29.7 & & \\
\hline 1.09 & 1.02 & 1.16 & 1.28 & 1.07 & \\
\hline 30.8 & 42.0 & 31.4 & 12.3 & 12.8 & \\
\hline 0.92 & 1.10 & 1.12 & 0.83 & 0.62 & \\
\hline 37.2 & 0.0 & 10.9 & 32.6 & 0.0 & \\
\hline 0.80 & 0.88 & 0.73 & 0.48 & & \\
\hline 29.7 & 11.1 & 0.0 & 0.0 & & \\
\hline 0.50 & 0.43 & & & & \\
\hline 0.0 & 0.0 & & & & \\
\hline
\end{tabular}

\begin{tabular}{|c|c|c|c|c|c|}
\hline 0.80 & & & & & \\
\hline 55.1 & & & & EOC $1 / 8$ & 8-Core $\mathrm{UO}_{2}$ \\
\hline 0.93 & 0.87 & & & $\mathrm{FeCrAl}($ & $(0.419 \mathrm{~mm})$ \\
\hline 39.7 & 56.4 & & & 1.30 & Relative power \\
\hline 0.89 & 1.20 & 0.94 & & 21.4 & Burn-up (GWd/t) \\
\hline 58.3 & 22.0 & 55.0 & & & \\
\hline 1.01 & 1.06 & 1.00 & 0.97 & & \\
\hline 41.2 & 36.8 & 41.7 & 48.0 & & \\
\hline 0.93 & 0.89 & 0.97 & 1.10 & 1.04 & \\
\hline 47.8 & 58.1 & 49.1 & 32.1 & 30.4 & \\
\hline 0.98 & 1.30 & 1.19 & 0.89 & 0.81 & \\
\hline 53.7 & 21.4 & 31.0 & 47.0 & 12.0 & \\
\hline 1.03 & 1.16 & 1.10 & 0.77 & & \\
\hline 45.8 & 29.3 & 16.1 & 10.7 & & \\
\hline 0.94 & 0.82 & & & & \\
\hline 12.5 & 10.8 & & & & \\
\hline
\end{tabular}

Figure 11. Radial power and burn-up distribution for $\mathrm{UO}_{2}$ fuel with FeCrAl $(0.419 \mathrm{~mm})$ cladding at BOC (left) and EOC (right). 


\begin{tabular}{|c|c|c|c|c|c|}
\hline 1.07 & & & & & \\
\hline 37.9 & & & & BOC $1 / 8$ & 8-Core $\mathrm{UO}_{2}$ \\
\hline 1.23 & 1.10 & & & FeCrAl & $(0.300 \mathrm{~mm})$ \\
\hline 20.6 & 37.9 & & & 1.39 & Relative power \\
\hline 1.13 & 1.33 & 1.22 & & 15.3 & Burn-up (GWd/t) \\
\hline 39.4 & 0.0 & 35.1 & & & \\
\hline 1.33 & 1.39 & 1.35 & 1.29 & & \\
\hline 20.6 & 15.3 & 21.3 & 27.6 & & \\
\hline 1.11 & 1.04 & 1.17 & 1.29 & 1.07 & \\
\hline 28.8 & 40.0 & 29.4 & 11.5 & 11.8 & \\
\hline 0.93 & 1.08 & 1.11 & 0.83 & 0.60 & \\
\hline 35.1 & 0.0 & 10.1 & 30.7 & 0.0 & \\
\hline 0.80 & 0.87 & 0.70 & 0.47 & & \\
\hline 27.6 & 10.2 & 0.0 & 0.0 & & \\
\hline 0.48 & 0.41 & & & & \\
\hline 0.0 & 0.0 & & & & \\
\hline
\end{tabular}

\begin{tabular}{|c|c|c|c|c|c|}
\hline 0.81 & & & & \multirow{2}{*}{\multicolumn{2}{|c|}{ EOC 1/8-Core $\mathrm{UO}_{2}$}} \\
\hline 52.5 & & & & & \\
\hline 0.94 & 0.88 & & & FeCrAl ( & $(0.300 \mathrm{~mm})$ \\
\hline 37.6 & 53.7 & & & 1.30 & Relative power \\
\hline 0.90 & 1.20 & 0.95 & & 20.1 & Burn-up (GWd/t) \\
\hline 55.4 & 20.9 & 52.2 & & & \\
\hline 1.01 & 1.07 & 1.01 & 0.98 & & \\
\hline 39.0 & 34.7 & 39.6 & 45.1 & & \\
\hline 0.95 & 0.90 & 0.98 & 1.10 & 1.03 & \\
\hline 45.1 & 55.3 & 46.3 & 30.3 & 28.4 & \\
\hline 0.99 & 1.30 & 1.19 & 0.89 & 0.80 & \\
\hline 50.8 & 20.1 & 28.9 & 44.3 & 11.2 & \\
\hline 1.03 & 1.16 & 1.09 & 0.75 & & \\
\hline 42.8 & 27.2 & 14.9 & 9.8 & & \\
\hline 0.93 & 0.81 & & & & \\
\hline 11.5 & 9.9 & & & & \\
\hline
\end{tabular}

Figure 12. Radial power and burn-up distribution for $\mathrm{UO}_{2}$ fuel with FeCrAl $(0.300 \mathrm{~mm})$ cladding at BOC (left) and EOC (right).

The approach in this paper is to develop reasonably representative core models, which will qualitatively inform the potential impacts of the new cladding materials in the PCMI stage of RIA progression. Burn-up limits of the cladding, irradiation behavior, and many other behaviors are not well known for these materials and are not considered in this scoping reactor physics analysis with thermal and hydraulic feedback. Each of these core calculations indicated relatively similar parameters throughout normal operation. The reactivity coefficients and kinetics parameters at an HZP condition at the beginning of the cycle are shown in Table 3. The parameters shown in Table 3 are fuel temperature coefficient (FTC), neutron generation time $(\Lambda)$ and delayed neutron fraction $\left(\beta_{\text {eff }}\right)$. 
Table 3. BOC HZP reactivity coefficients and kinetics parameters.

\begin{tabular}{llllll}
\hline Cladding material & Zircaloy & SiC/SiC & SiC/SiC & FeCrAl & FeCrAl \\
\hline Cladding thickness $(\mathbf{m m})$ & 0.572 & 0.572 & $\mathbf{0 . 7 6 2}$ & $\mathbf{0 . 4 1 9}$ & $\mathbf{0 . 3 0 0}$ \\
$\mathbf{F T C}(\mathbf{\$} / \mathbf{K})$ & $-4.7 \mathrm{E}-03$ & $-4.7 \mathrm{E}-03$ & $-4.5 \mathrm{E}-03$ & $-4.7 \mathrm{E}-03$ & $-4.8 \mathrm{E}-03$ \\
$\boldsymbol{\Lambda}(\mathbf{s})$ & $1.44 \mathrm{E}-05$ & $1.43 \mathrm{E}-05$ & $1.62 \mathrm{E}-05$ & $1.15 \mathrm{E}-05$ & $1.16 \mathrm{E}-05$ \\
$\boldsymbol{\beta}_{\text {eff }}$ & 0.0060 & 0.0060 & 0.0060 & 0.0061 & 0.0061 \\
\hline
\end{tabular}

\section{Comparison of Assembly Few-Group Parameters using SCALE 6.1 (TRITON/NEWT) and}

\section{SCALE 6.2 (Polaris) and Reactor Core Kinetics Parameter Results}

As discussed in Section 2, the lattice physics for the few-group parameters in this paper uses SCALE 6.1 with the TRITON/NEWT lattice physics capability, ENDF/B-VII.0 cross sections, and a simplified set of four burnable materials in the lattice physics modeling. These assumptions are consistent with the methodologies established in previous scoping analyses of ATF concepts (Brown et al. 2013, Brown et al. 2014, Brown et al. 2015, George et al. 2015, Todosow et al. 2015).

SCALE 6.2 includes a new lattice physics capability, Polaris, a two-dimensional method-of-characteristics neutron transport solver optimized for LWR lattice physics, which uses the embedded self-shielding method (Jessee et al. 2014). To identify potential sensitivities to the selection of lattice physics tool, nuclear data library, and modeling simplifications, the TRITON/NEWT lattice physics cases used to generate the core models in Section 2 were re-generated in the Polaris lattice physics tool. The specific differences between the lattice physics models compared are:

- Use of the new LWR optimized Polaris advanced lattice physics capability and self-shielding methodology in SCALE 6.2 versus the general geometry TRITON/NEWT lattice physics capability in SCALE 6.1, 
- ENDF/B-VII.1 nuclear data libraries in 56 groups versus ENDF/B-VII.0 data in 49 groups, and

- Explicit depletion of each fuel pin (fuel and cladding) material versus a simplified set of burnable fuel and cladding materials.

The objective of this Section of the paper is to investigate potential differences in the lattice physics and core modeling results due to the use of different modeling methods, assumptions, and underlying data. Lattice physics models were generated using Polaris for the configurations identified in Table 1. A comparison of the assembly beginning-of-life infinite multiplication factor and the difference in percent millirho $(\mathrm{pcm})$ is shown in Table 4. The results are in reasonable agreement, with the Polaris results for Zircaloy and $\mathrm{SiC} / \mathrm{SiC}$ cladding showing a difference of approximately 200 pcm. Although not shown, the difference in the assembly infinite multiplication factor results as a function of fuel depletion is similar, with a maximum bias of approximately $250 \mathrm{pcm}$. The differences Table 4 are driven by differences in reactor physics methods, cross section libraries and group structure, and underlying nuclear data.

Table 4. Comparison of assembly beginning-of-life infinite multiplication factor.

\begin{tabular}{cccc}
\hline Cladding material / thickness (mm) & TRITON/NEWT & Polaris & difference (pcm) \\
\hline Zircaloy / 0.572 & 1.10924 & 1.10731 & 192 \\
SiC/SiC / 0.572 & 1.11538 & 1.11316 & 222 \\
SiC/SiC / 0.762 & 1.10978 & 1.10728 & 250 \\
FeCrAl / 0.419 & 1.09948 & 1.09991 & -44 \\
FeCrAl / 0.300 & 1.08437 & 1.08366 & 71 \\
\hline
\end{tabular}


A comparison of few-group parameters used in the core calculation is shown in Table 5. Group 1 is the fast group and group 2 is the thermal group. All of the few-group parameters show good agreement, with some cross sections (such as fast group transport) showing a small but consistent bias across the cases.

Table 5. Comparison of homogenized two-group parameters for a reflected assembly at beginning-of-life. The SCALE 6.1 results use ENDF/B-VII.0 data and SCALE 6.2 results use ENDF/B-VII.1 data.

\begin{tabular}{|c|c|c|c|c|c|c|}
\hline \multicolumn{2}{|r|}{ Cross sections } & \multicolumn{5}{|c|}{ Cladding material / thickness (mm) } \\
\hline \multirow{5}{*}{$\begin{array}{c}\text { Transport } \\
\text { (g1) }\end{array}$} & & Zircaloy / & $\mathrm{SiC} / \mathrm{SiC} /$ & $\mathrm{SiC} / \mathrm{SiC} /$ & $\mathrm{FeCrAl} /$ & $\mathrm{FeCrAl} /$ \\
\hline & & $0.572 \mathrm{~mm}$ & $0.572 \mathrm{~mm}$ & $0.762 \mathrm{~mm}$ & $0.419 \mathrm{~mm}$ & $0.300 \mathrm{~mm}$ \\
\hline & SCALE 6.1 (TRITON/NEWT) & $2.240 \mathrm{E}-01$ & $2.263 \mathrm{E}-01$ & $2.255 \mathrm{E}-01$ & $2.318 \mathrm{E}-01$ & $2.316 \mathrm{E}-01$ \\
\hline & SCALE 6.2 (Polaris) & 2.152E-01 & 2.169E-01 & 2.157E-01 & 2.202E-01 & $2.208 \mathrm{E}-01$ \\
\hline & Difference $(\%)$ & 4.0 & 4.3 & 4.4 & 5.1 & 4.8 \\
\hline \multirow{3}{*}{$\begin{array}{c}\text { Transport } \\
\text { (g2) }\end{array}$} & SCALE 6.1 (TRITON/NEWT) & $9.097 \mathrm{E}-01$ & $9.121 \mathrm{E}-01$ & $9.067 \mathrm{E}-01$ & $9.551 \mathrm{E}-01$ & $9.466 \mathrm{E}-01$ \\
\hline & SCALE 6.2 (Polaris) & 8.987E-01 & $9.010 \mathrm{E}-01$ & 8.959E-01 & $9.437 \mathrm{E}-01$ & $9.352 \mathrm{E}-01$ \\
\hline & Difference $(\%)$ & 1.2 & 1.2 & 1.2 & 1.2 & 1.2 \\
\hline \multirow{3}{*}{$\begin{array}{l}\text { Absorption } \\
\text { (g1) }\end{array}$} & SCALE 6.1 (TRITON/NEWT) & $1.161 \mathrm{E}-02$ & $1.152 \mathrm{E}-02$ & 1.079E-02 & $1.270 \mathrm{E}-02$ & $1.284 \mathrm{E}-02$ \\
\hline & SCALE 6.2 (Polaris) & 1.139E-02 & $1.132 \mathrm{E}-02$ & 1.059E-02 & $1.240 \mathrm{E}-02$ & $1.256 \mathrm{E}-02$ \\
\hline & Difference $(\%)$ & 1.9 & 1.8 & 1.9 & 2.4 & 2.2 \\
\hline \multirow{3}{*}{$\begin{array}{c}\text { Absorption } \\
\text { (g2) }\end{array}$} & SCALE 6.1 (TRITON/NEWT) & $1.390 \mathrm{E}-01$ & $1.391 \mathrm{E}-01$ & $1.301 \mathrm{E}-01$ & $1.586 \mathrm{E}-01$ & $1.564 \mathrm{E}-01$ \\
\hline & SCALE 6.2 (Polaris) & $1.386 \mathrm{E}-01$ & $1.387 \mathrm{E}-01$ & $1.298 \mathrm{E}-01$ & $1.580 \mathrm{E}-01$ & $1.559 \mathrm{E}-01$ \\
\hline & Difference $(\%)$ & 0.3 & 0.3 & 0.3 & 0.3 & 0.4 \\
\hline \multirow{3}{*}{$\begin{array}{c}\text { Nu*fission } \\
\text { (g1) }\end{array}$} & SCALE 6.1 (TRITON/NEWT) & $9.171 \mathrm{E}-03$ & $9.213 \mathrm{E}-03$ & 8.487E-03 & $1.038 \mathrm{E}-02$ & $1.026 \mathrm{E}-02$ \\
\hline & SCALE 6.2 (Polaris) & $9.017 \mathrm{E}-03$ & $9.059 \mathrm{E}-03$ & 8.343E-03 & $1.020 \mathrm{E}-02$ & $1.008 \mathrm{E}-02$ \\
\hline & Difference $(\%)$ & 1.7 & 1.7 & 1.7 & 1.8 & 1.7 \\
\hline \multirow{3}{*}{$\begin{array}{c}\text { Nu*fission } \\
\text { (g2) }\end{array}$} & SCALE 6.1 (TRITON/NEWT) & $1.882 \mathrm{E}-01$ & $1.884 \mathrm{E}-01$ & $1.735 \mathrm{E}-01$ & 2.133E-01 & $2.087 \mathrm{E}-01$ \\
\hline & SCALE 6.2 (Polaris) & $1.868 \mathrm{E}-01$ & $1.869 \mathrm{E}-01$ & $1.721 \mathrm{E}-01$ & $2.114 \mathrm{E}-01$ & $2.068 \mathrm{E}-01$ \\
\hline & Difference $(\%)$ & 0.8 & 0.8 & 0.8 & 0.9 & 0.9 \\
\hline
\end{tabular}




\begin{tabular}{clccccc}
\hline Scattering & SCALE 6.1 (TRITON/NEWT) & $1.540 \mathrm{E}-02$ & $1.548 \mathrm{E}-02$ & $1.591 \mathrm{E}-02$ & $1.486 \mathrm{E}-02$ & $1.490 \mathrm{E}-02$ \\
(g1 to g2) & SCALE 6.2 (Polaris) & $1.523 \mathrm{E}-02$ & $1.533 \mathrm{E}-02$ & $1.575 \mathrm{E}-02$ & $1.472 \mathrm{E}-02$ & $1.476 \mathrm{E}-02$ \\
& Difference (\%) & 1.1 & 1.0 & 1.0 & 0.9 & 1.0 \\
Scattering & SCALE 6.1 (TRITON/NEWT) & $2.462 \mathrm{E}-03$ & $2.486 \mathrm{E}-03$ & $2.325 \mathrm{E}-03$ & $2.929 \mathrm{E}-03$ & $2.882 \mathrm{E}-03$ \\
(g2 to g1) & SCALE 6.2 (Polaris) & $2.392 \mathrm{E}-03$ & $2.416 \mathrm{E}-03$ & $2.264 \mathrm{E}-03$ & $2.841 \mathrm{E}-03$ & $2.795 \mathrm{E}-03$ \\
& Difference (\%) & 2.9 & 2.8 & 2.6 & 3.0 & 3.1 \\
\multirow{2}{*}{ ADF (g1) } & SCALE 6.1 (TRITON/NEWT) & $1.018 \mathrm{E}+00$ & $1.018 \mathrm{E}+00$ & $1.018 \mathrm{E}+00$ & $1.019 \mathrm{E}+00$ & $1.019 \mathrm{E}+00$ \\
& SCALE 6.2 (Polaris) & $1.003 \mathrm{E}+00$ & $1.003 \mathrm{E}+00$ & $1.002 \mathrm{E}+00$ & $1.003 \mathrm{E}+00$ & $1.004 \mathrm{E}+00$ \\
& Difference (\%) & 1.5 & 1.5 & 1.6 & 1.5 & 1.5 \\
ADF (g2) & SCALE 6.1 (TRITON/NEWT) & $1.087 \mathrm{E}+00$ & $1.087 \mathrm{E}+00$ & $1.083 \mathrm{E}+00$ & $1.097 \mathrm{E}+00$ & $1.099 \mathrm{E}+00$ \\
& SCALE 6.2 (Polaris) & $1.093 \mathrm{E}+00$ & $1.093 \mathrm{E}+00$ & $1.086 \mathrm{E}+00$ & $1.105 \mathrm{E}+00$ & $1.105 \mathrm{E}+00$ \\
& Difference (\%) & -0.5 & -0.5 & -0.3 & -0.7 & -0.5 \\
\hline
\end{tabular}

The equilibrium core kinetics parameters, which govern the response to the RIA transient, were also compared in Table 6. The fuel temperature coefficient, neutron generation time, delayed neutron fraction, radial assembly power peaking, and local power peaking are shown. These parameters are shown at Hot Full Power (HFP) conditions at BOC and EOC. The results shown good agreement for both integral kinetics parameters and local power peaking.

Table 6. Comparison of integral and local results from PARCS core calculations using two sets of few-group parameters.

\begin{tabular}{|c|c|c|c|c|c|c|}
\hline \multicolumn{2}{|c|}{ PARCS Core Calculation at HFP } & \multicolumn{5}{|c|}{ Cladding material / thickness (mm) } \\
\hline & & Zircaloy / & $\mathrm{SiC} / \mathrm{SiC} /$ & $\mathrm{SiC} / \mathrm{SiC} /$ & $\mathrm{FeCrAl} /$ & $\mathrm{FeCrAl} /$ \\
\hline & & $0.572 \mathrm{~mm}$ & $0.572 \mathrm{~mm}$ & $0.762 \mathrm{~mm}$ & $0.419 \mathrm{~mm}$ & $0.300 \mathrm{~mm}$ \\
\hline \multirow{2}{*}{$\begin{array}{c}\text { FTC }(\$ / K) \\
\text { at BOC }\end{array}$} & SCALE 6.1 (TRITON/NEWT) & $-4.708 \mathrm{E}-03$ & $-4.653 \mathrm{E}-03$ & $-4.411 \mathrm{E}-03$ & $-4.618 \mathrm{E}-03$ & $-4.806 \mathrm{E}-03$ \\
\hline & SCALE 6.2 (Polaris) & $-4.561 \mathrm{E}-03$ & $-4.346 \mathrm{E}-03$ & $-4.086 \mathrm{E}-03$ & $-4.465 \mathrm{E}-03$ & $-4.687 \mathrm{E}-03$ \\
\hline
\end{tabular}




\begin{tabular}{|c|c|c|c|c|c|c|}
\hline & Difference $(\%)$ & 3.2 & 6.8 & 7.7 & 3.4 & 2.5 \\
\hline \multirow{3}{*}{$\begin{array}{c}\text { FTC }(\$ / K) \\
\text { at EOC }\end{array}$} & SCALE 6.1 (TRITON/NEWT) & $-5.611 \mathrm{E}-03$ & $-5.540 \mathrm{E}-03$ & $-5.351 \mathrm{E}-03$ & $-5.509 \mathrm{E}-03$ & $-5.717 \mathrm{E}-03$ \\
\hline & SCALE 6.2 (Polaris) & $-5.575 \mathrm{E}-03$ & $-5.321 \mathrm{E}-03$ & $-5.029 \mathrm{E}-03$ & $-5.488 \mathrm{E}-03$ & $-5.746 \mathrm{E}-03$ \\
\hline & Difference $(\%)$ & 0.6 & 4.0 & 6.2 & 0.4 & -0.5 \\
\hline \multirow{3}{*}{$\begin{array}{c}\Lambda(s) \text { at } \\
\text { BOC }\end{array}$} & SCALE 6.1 (TRITON/NEWT) & $1.466 \mathrm{E}-05$ & $1.458 \mathrm{E}-05$ & $1.663 \mathrm{E}-05$ & $1.157 \mathrm{E}-05$ & 1.167E-05 \\
\hline & SCALE 6.2 (Polaris) & $1.498 \mathrm{E}-05$ & $1.491 \mathrm{E}-05$ & $1.704 \mathrm{E}-05$ & $1.179 \mathrm{E}-05$ & $1.189 \mathrm{E}-05$ \\
\hline & Difference $(\%)$ & -2.2 & -2.3 & -2.4 & -1.9 & -1.9 \\
\hline \multirow{3}{*}{$\begin{array}{c}\Lambda \text { (s) at } \\
\text { EOC }\end{array}$} & SCALE 6.1 (TRITON/NEWT) & $1.762 \mathrm{E}-05$ & $1.758 \mathrm{E}-05$ & $2.049 \mathrm{E}-05$ & $1.342 \mathrm{E}-05$ & $1.332 \mathrm{E}-05$ \\
\hline & SCALE 6.2 (Polaris) & $1.788 \mathrm{E}-05$ & $1.786 \mathrm{E}-05$ & $2.085 \mathrm{E}-05$ & $1.362 \mathrm{E}-05$ & $1.352 \mathrm{E}-05$ \\
\hline & Difference (\%) & -1.5 & -1.6 & -1.8 & -1.5 & -1.5 \\
\hline \multirow[t]{3}{*}{$\beta_{\text {eff }}$ at BOC } & SCALE 6.1 (TRITON/NEWT) & 0.0060 & 0.0060 & 0.0059 & 0.0061 & 0.0061 \\
\hline & SCALE 6.2 (Polaris) & 0.0057 & 0.0057 & 0.0057 & 0.0058 & 0.0058 \\
\hline & Difference $(\%)$ & 4.9 & 4.9 & 4.5 & 5.3 & 5.6 \\
\hline \multirow[t]{3}{*}{$\beta_{\text {eff }}$ at EOC } & SCALE 6.1 (TRITON/NEWT) & 0.0053 & 0.0054 & 0.0053 & 0.0055 & 0.0055 \\
\hline & SCALE 6.2 (Polaris) & 0.0051 & 0.0051 & 0.0050 & 0.0052 & 0.0052 \\
\hline & Difference (\%) & 5.5 & 5.5 & 5.1 & 5.8 & 6.2 \\
\hline \multirow[t]{3}{*}{$\mathrm{F}_{\mathrm{xy}}$ at BOC } & SCALE 6.1 (TRITON/NEWT) & 1.36 & 1.36 & 1.37 & 1.38 & 1.39 \\
\hline & SCALE 6.2 (Polaris) & 1.37 & 1.37 & 1.38 & 1.39 & 1.40 \\
\hline & Difference $(\%)$ & -0.5 & -0.6 & -0.4 & -0.7 & -0.6 \\
\hline \multirow[t]{3}{*}{$F_{x y}$ at $\mathrm{EOC}$} & SCALE 6.1 (TRITON/NEWT) & 1.33 & 1.33 & 1.34 & 1.30 & 1.30 \\
\hline & SCALE 6.2 (Polaris) & 1.33 & 1.33 & 1.34 & 1.30 & 1.30 \\
\hline & Difference (\%) & 0.0 & 0.0 & 0.0 & 0.0 & 0.0 \\
\hline \multirow[t]{3}{*}{$\mathrm{F}_{\mathrm{xyz}}$ at BOC } & SCALE 6.1 (TRITON/NEWT) & 1.56 & 1.54 & 1.57 & 1.59 & 1.63 \\
\hline & SCALE 6.2 (Polaris) & 1.56 & 1.55 & 1.58 & 1.60 & 1.63 \\
\hline & Difference $(\%)$ & 0.0 & -0.5 & -0.2 & -0.4 & 0.0 \\
\hline \multirow[t]{3}{*}{$F_{\mathrm{xyz}}$ at EOC } & SCALE 6.1 (TRITON/NEWT) & 1.53 & 1.54 & 1.49 & 1.51 & 1.50 \\
\hline & SCALE 6.2 (Polaris) & 1.52 & 1.52 & 1.49 & 1.50 & 1.49 \\
\hline & Difference $(\%)$ & 1.2 & 1.1 & 0.0 & 0.8 & 0.8 \\
\hline
\end{tabular}

These comparisons show good agreement between few-group parameter and core calculation results using two different lattice physics tools, nuclear data libraries, and modeling simplifications. 
To maintain consistency with prior efforts (Brown et al. 2013, Brown et al. 2014, Brown et al. 2015, George et al. 2015, Todosow et al. 2015), the few-group parameters generated using SCALE 6.1 (TRITON/NEWT) with ENDF/B-VII.0 cross sections and a simplified set of depletion materials were utilized in the reactor core kinetics calculations described in Section 5.

\section{Reactivity Initiated Accident Simulation}

Three sets of HZP RIA cases were considered and simulated using the PARCS three-dimensional nodal kinetics capability for a total of fifteen simulated scenarios. The first set of cases (Case 1) considers a comparison in which each case has a similar reactivity worth of approximately $\$ 1.74$. The selected super prompt reactivity worth is very similar to other relevant studies, including Hursin et al. (2013) and Andrews et al. (2016). In particular, Hursin et al. (2013) is a relevant study due to its focus on the coupled neutronics and fuel performance aspects of RIA. In this case, the inserted reactivity is imposed instantaneously upon the reactor core kinetics model via ejection of a multi-cluster control rod bank. The ejected rod height is slightly adjusted in these cases to yield the same total worth for each rod ejection.

The second set of cases (Case 2) considers the important fact that the different cladding materials yield different control rod worth for the same control rod material. In this case, the same multi-cluster instantaneous ejection is performed, but with a consistent ejection for all cases. This yields slightly different reactivity worth for all cases.

The third set of cases (Case 3) is the same as the second case, but instead of an instantaneous ejection, the ejection occurs over a time delay of 0.1 seconds. Thus, the reactivity insertion rate is different for each of the cases due to the different total rod worth. 


\subsection{Results for Case 1: Similar Total Reactivity Worth, Instantaneous Ejection}

A super-prompt critical RIA was simulated for each of the analyzed configurations shown in Table 3. The RIA considered in this paper was an instantaneous step reactivity insertion worth approximately $\$ 1.74$. The PARCS 3-D nodal kinetics solution was used with default transient convergence criteria settings. The reactivity insertion is shown in Figure 13, and the power pulse response is shown in Figure 14. The corresponding increase in maximum fuel temperature, averaged across the assembly for the peak axial location in the core, is shown in Figure 15. A constant gas gap conductance of 5,000 W/m-K was assumed for all cases. It is notable that different cladding materials would be expected to have different gap conductance due to heat transfer properties, creep, gap closure, and other phenomena. 


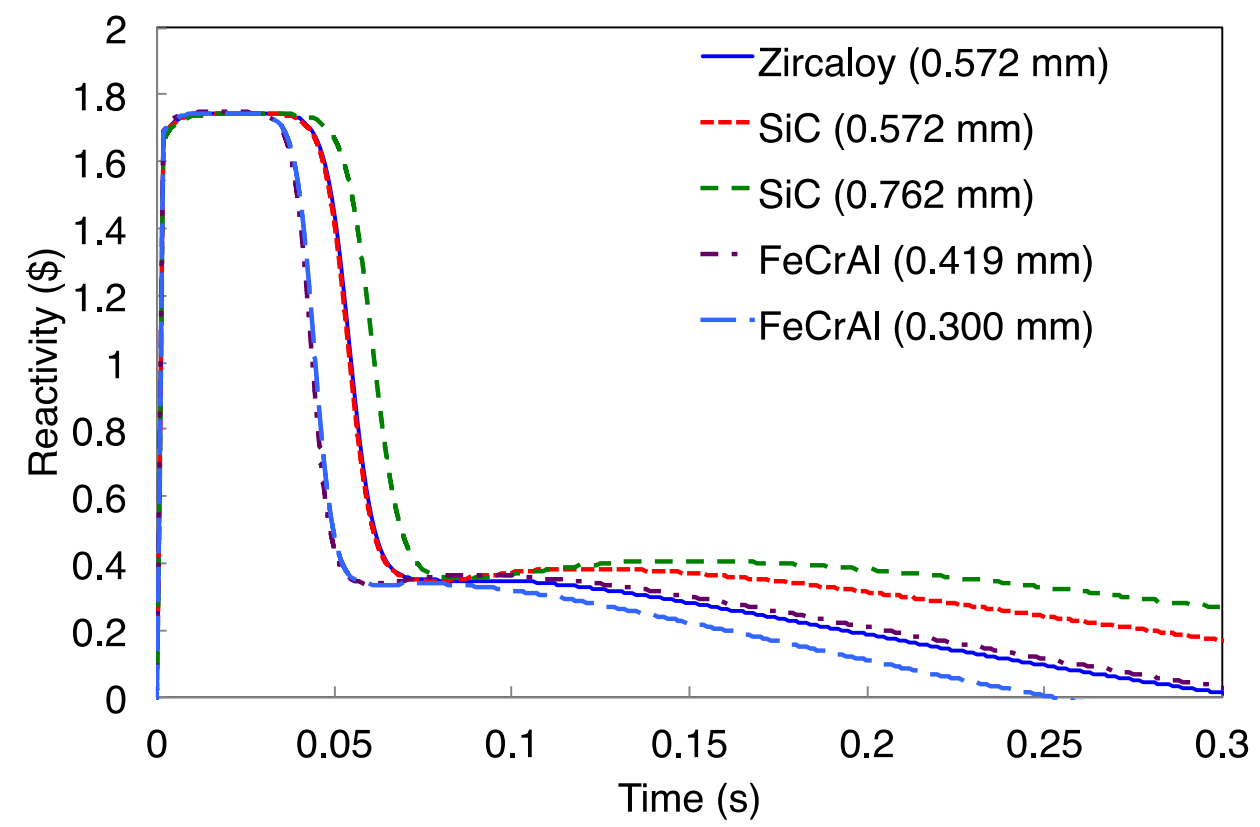

Figure 13. Super-prompt critical reactivity step insertion, Case 1. 


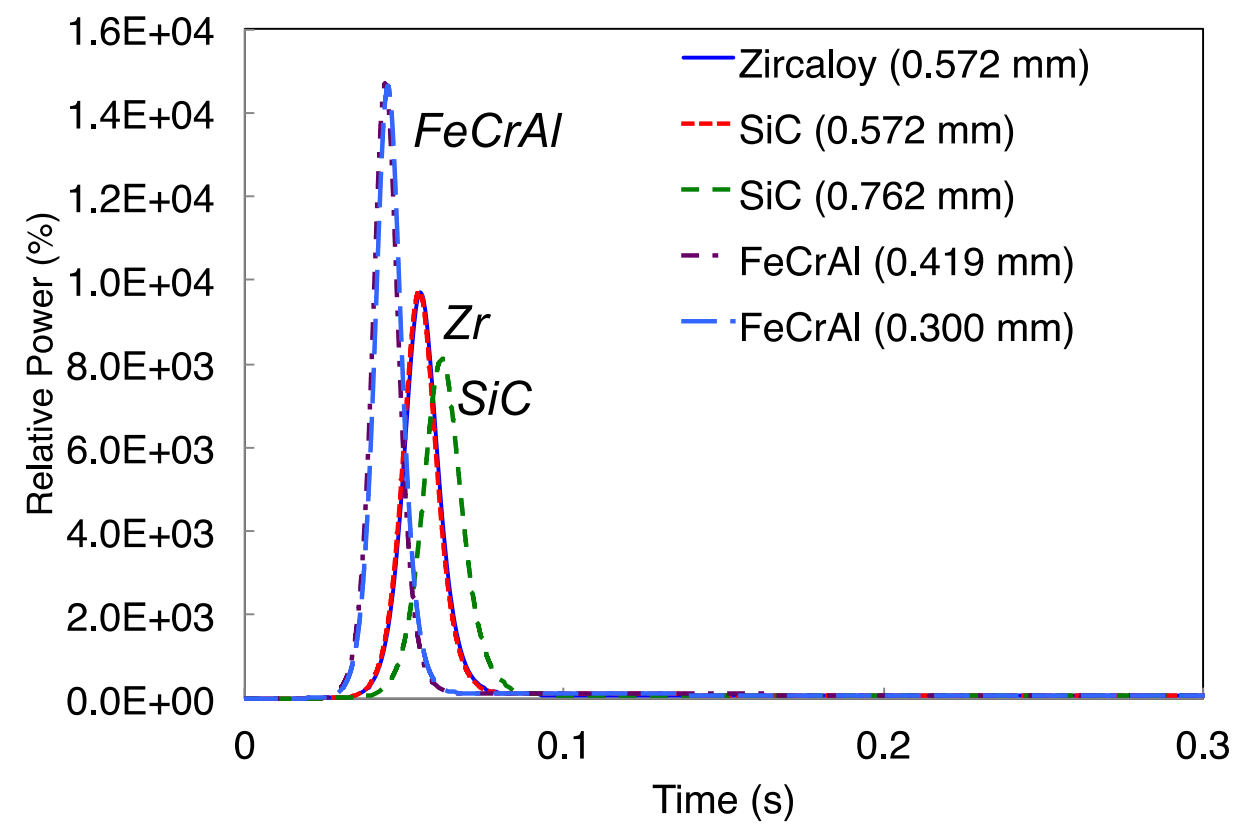

Figure 14. Super-prompt critical power response, Case 1. Note: Zircaloy and SiC/SiC results overlap at the $0.572 \mathrm{~mm}$ cladding thickness. 


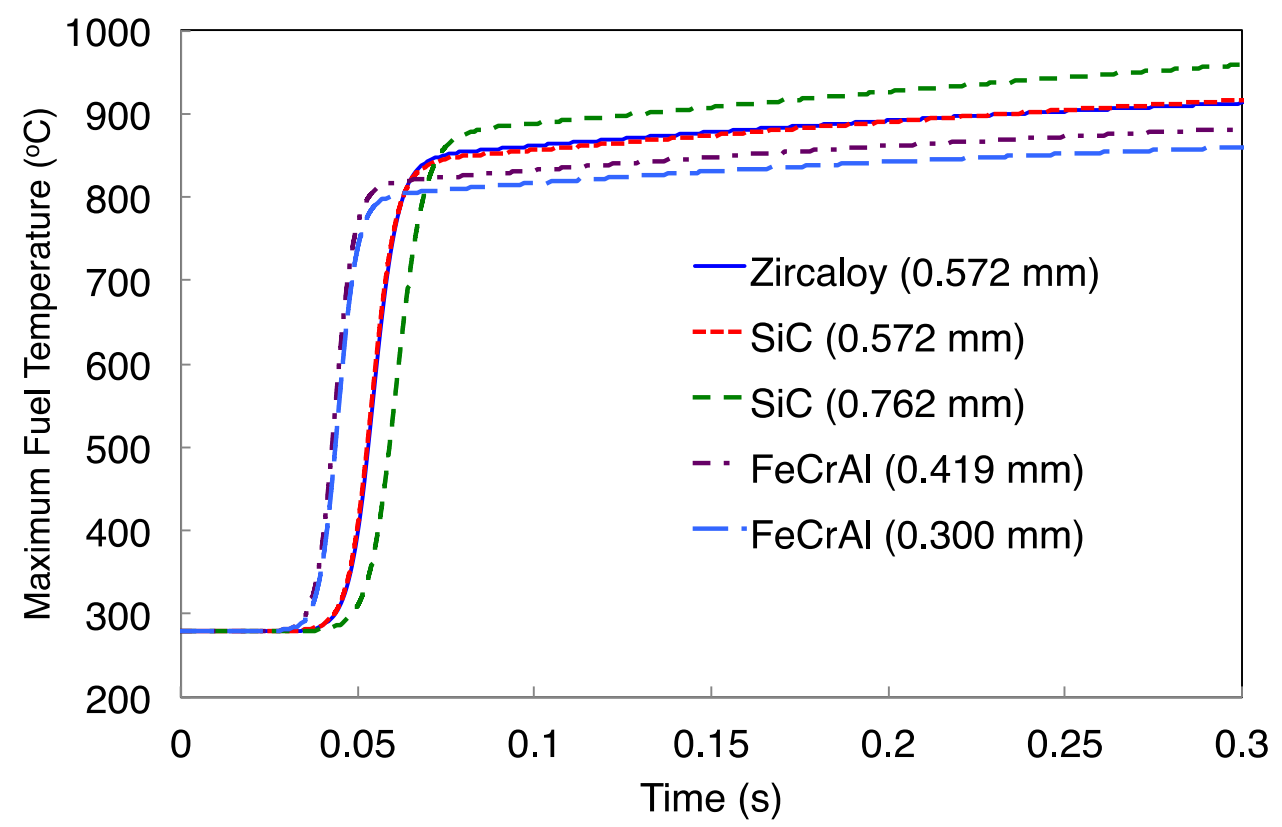

Figure 15. Super-prompt critical maximum fuel temperature increase, Case 1.

The main difference in response is the sharper pulse of shorter duration in the FeCrAl cladding cases. This characteristic reflects the relatively shorter neutron generation time of the hardened spectrum in these cases. The hardened spectrum is the result of the increase in fuel-to-moderator ratio and the parasitic absorption of thermal and epithermal neutrons in iron and chromium.

The important parameters to inform the PCMI separate effects experiment design include the fuel diameter change $(\%)$, full width at half maximum of the pulse, and fuel diameter change rate $(\% / s)$. The correlation for uranium dioxide thermal expansion from FRAPTRAN was used to estimate the fuel-diameter change during the transient (Luscher et al. 2011). This approach neglects the transient impacts of fission gas bubble expansion, but according to Sonnenburg (2012), these effects become 
most important at local energy depositions above $110 \mathrm{cal} / \mathrm{g}$, which is considerably higher than the highest local energy deposition calculated in this work $(58 \mathrm{cal} / \mathrm{g})$. The use of the MATPRO correlation for uranium dioxide thermal expansion to estimate the cladding strain is consistent with the NRC's transient fuel performance tool FRAPTRAN. The FRAPTRAN manual states (Luscher et al. 2011): "Once the fuel-cladding gap is closed, the cladding is assumed to follow the fuel dimensional changes from fuel thermal expansion. ... This may overpredict fuel-cladding mechanical interaction strain for some transients." This approximation neglects the mechanical resistance applied by the cladding during the RIA transient. In this work, expansion is assumed to be isotropic and there is no explicit consideration of when contact between the pellet and cladding occurs.

The estimated fuel thermal expansion during the transient is shown in Figure 16. The fuel thermal expansion rate, as shown in Figure 17, indicates that the FeCrAl cases expand faster than the Zircaloy or $\mathrm{SiC} / \mathrm{SiC}$ cases, due to the shorter neutron generation time of the $\mathrm{FeCrAl}$ cases. Experiments are necessary to determine whether the potential differences in strain rate result in differences in cladding material mechanical behavior, including failure. 


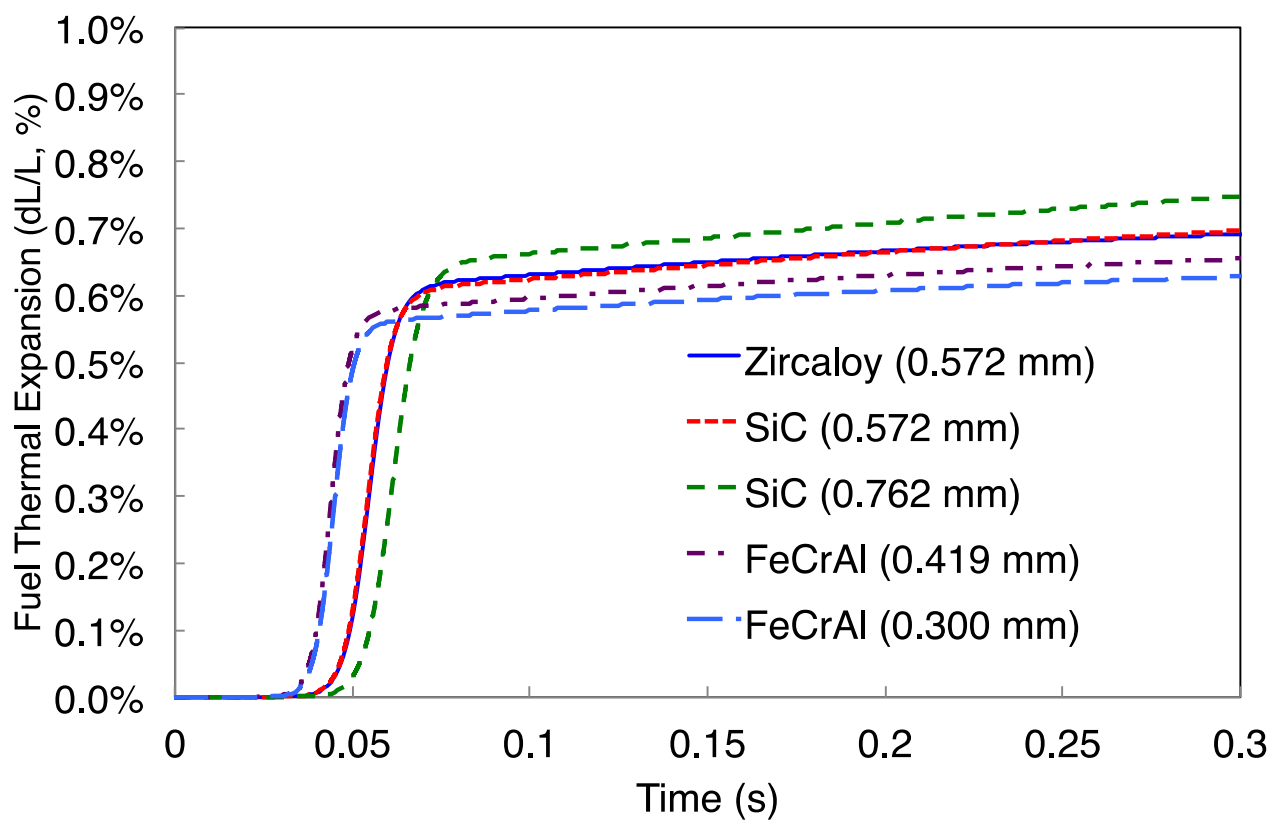

Figure 16. Super-prompt critical maximum unconstrained fuel thermal expansion (\%), Case 1. 


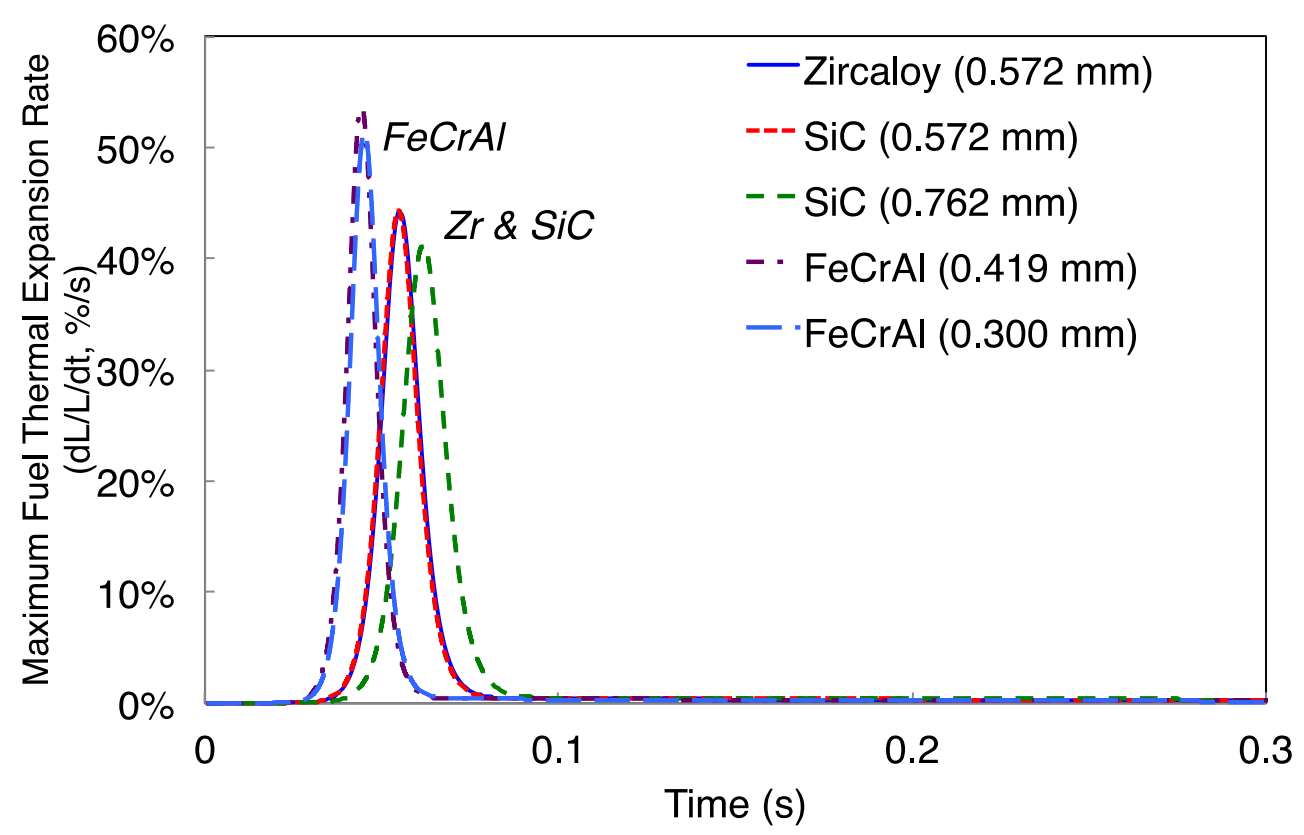

Figure 17. Super-prompt critical maximum unconstrained fuel thermal expansion rate (\%/s), Case 1.

The maximum energy deposition is similar for each of the transients in Figure 14, ranging from $46.9 \mathrm{cal} / \mathrm{g}$ to $57.6 \mathrm{cal} / \mathrm{g}$. Note that these cases assume ejection of an entire bank of control rod clusters and do not include pin power reconstruction, so this energy deposition is averaged over an assembly for a peak axial location. Although these assumptions spread the energy deposition of the power pulse throughout the reactor core-whereas a single high worth rod cluster ejection might be expected to inject more fuel enthalpy locally — these calculations are qualitatively informative because they identify relative reactor physics differences in the cases. A summary of the results is shown in Table 7. All of the results are relatively similar, but the FeCrAl cases feature shorter times to peak power, greater magnitudes of the power pulse, and shorter full widths at half maximum. The PARCS 
standalone simulations explicitly account for three-dimensional reactor kinetics and heat transfer to the cladding and coolant. To illustrate the non-Gaussian behavior, the power response is shown in Figure 18 with the vertical axis plotted on a logarithmic scale.

Table 7. Summary of integral super-prompt RIA results.

\begin{tabular}{llllll}
\hline Cladding material & Zircaloy & SiC/SiC & SiC/SiC & FeCrAl & FeCrAl \\
\hline Cladding thickness $(\mathbf{m m})$ & 0.572 & 0.572 & $\mathbf{0 . 7 6 2}$ & $\mathbf{0 . 4 1 9}$ & $\mathbf{0 . 3 0 0}$ \\
\hline Time of peak power $(\mathbf{m s})$ & 55.0 & 54.6 & 61.6 & 43.9 & 44.8 \\
\hline $\begin{array}{l}\text { Peak power }(\% \text { of nominal) } \\
\text { Full width at half maximum } \\
\text { (ms) }\end{array}$ & $9.71 \mathrm{E}+03$ & $9.74 \mathrm{E}+03$ & $8.11 \mathrm{E}+03$ & $1.47 \mathrm{E}+04$ & $1.46 \mathrm{E}+04$ \\
$\begin{array}{l}\text { Node average peak energy } \\
\text { deposition }(\mathbf{c a l} / \mathbf{g})\end{array}$ & 51.8 & 12.1 & 13.9 & 9.42 & 9.56 \\
\hline
\end{tabular}




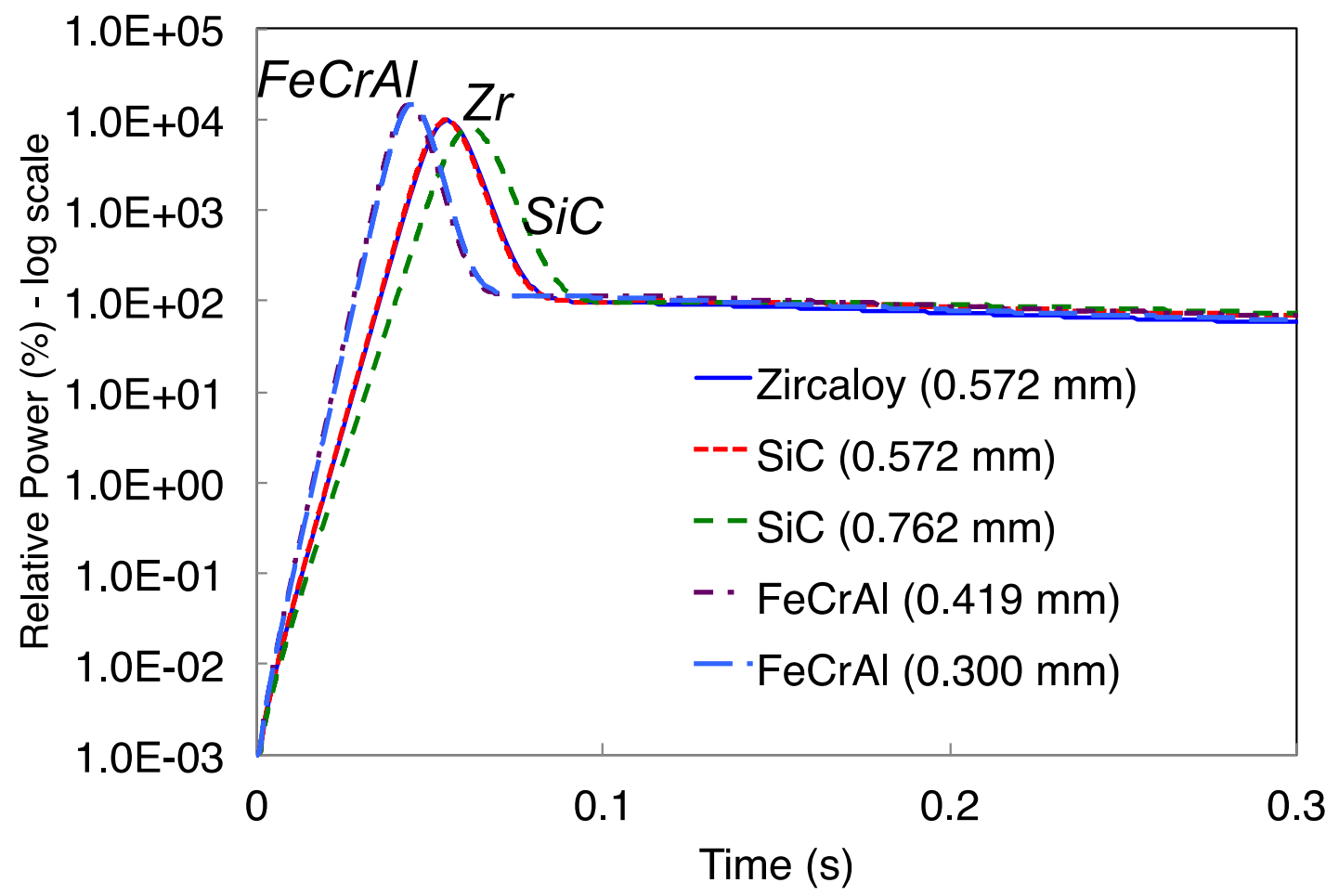

Figure 18. Super-prompt critical power response, log-scale on vertical axis, Case 1. Note: Zircaloy and $\mathrm{SiC} / \mathrm{SiC}$ results overlap at the $0.572 \mathrm{~mm}$ cladding thickness.

\subsection{Results for Case 2: Different Total Reactivity Worth, Instantaneous Ejection}

The spectral effects that result from the various cladding materials and associated lattice designs yield differences in control rod worth. These small differences impact the behavior of a super prompt RIA. In Case 2, the same multi-rod ejection is performed for all configurations presented in the paper. Unlike Case 1, the insertion of the ejected bank is not adjusted to give the same worth for each case. The reactivity insertions are shown in Figure 19, and the power pulse responses are shown in Figure 
20. The corresponding increase in maximum fuel temperature (averaged across the assembly for the peak axial location in the core) is shown in Figure 21. Although there are small differences in the ejected control rod worth, the general trends in behavior are similar to those of Case 1.

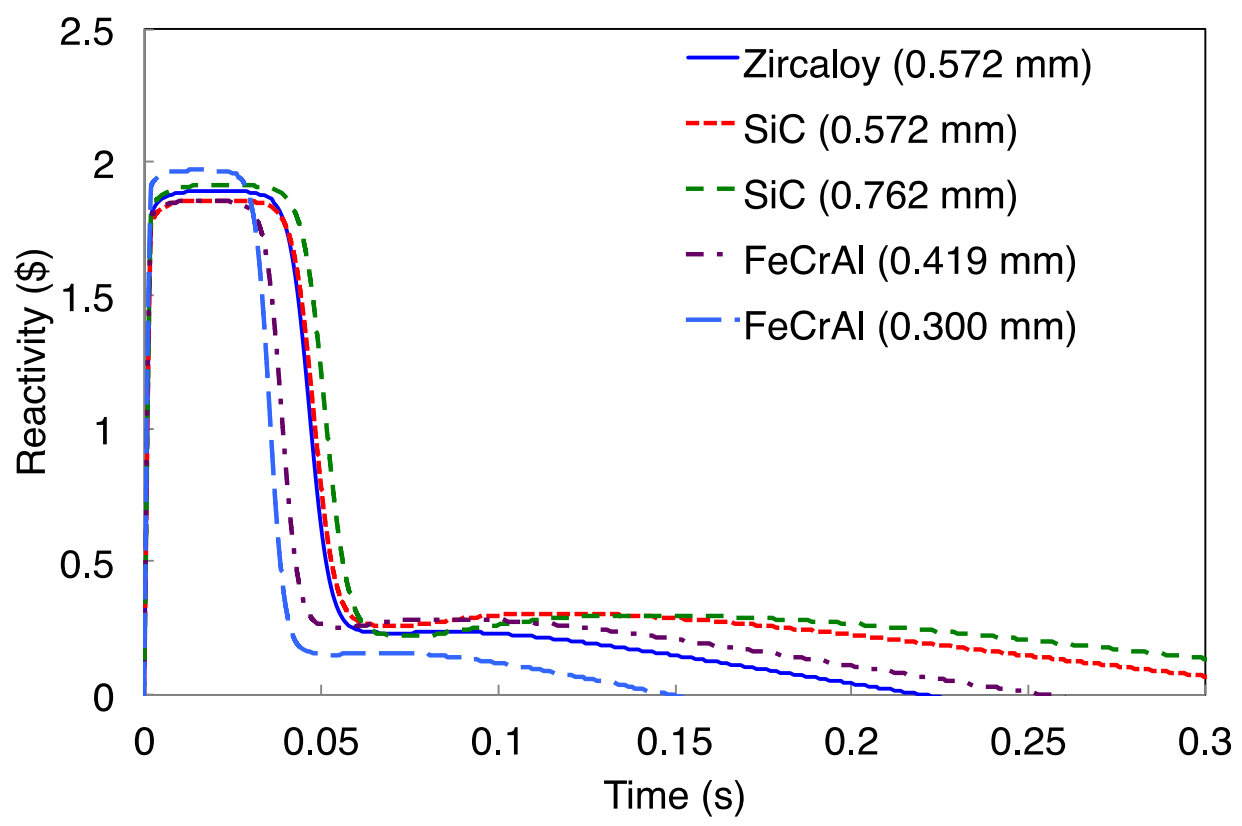

Figure 19. Super-prompt critical reactivity step insertion, Case 2. 


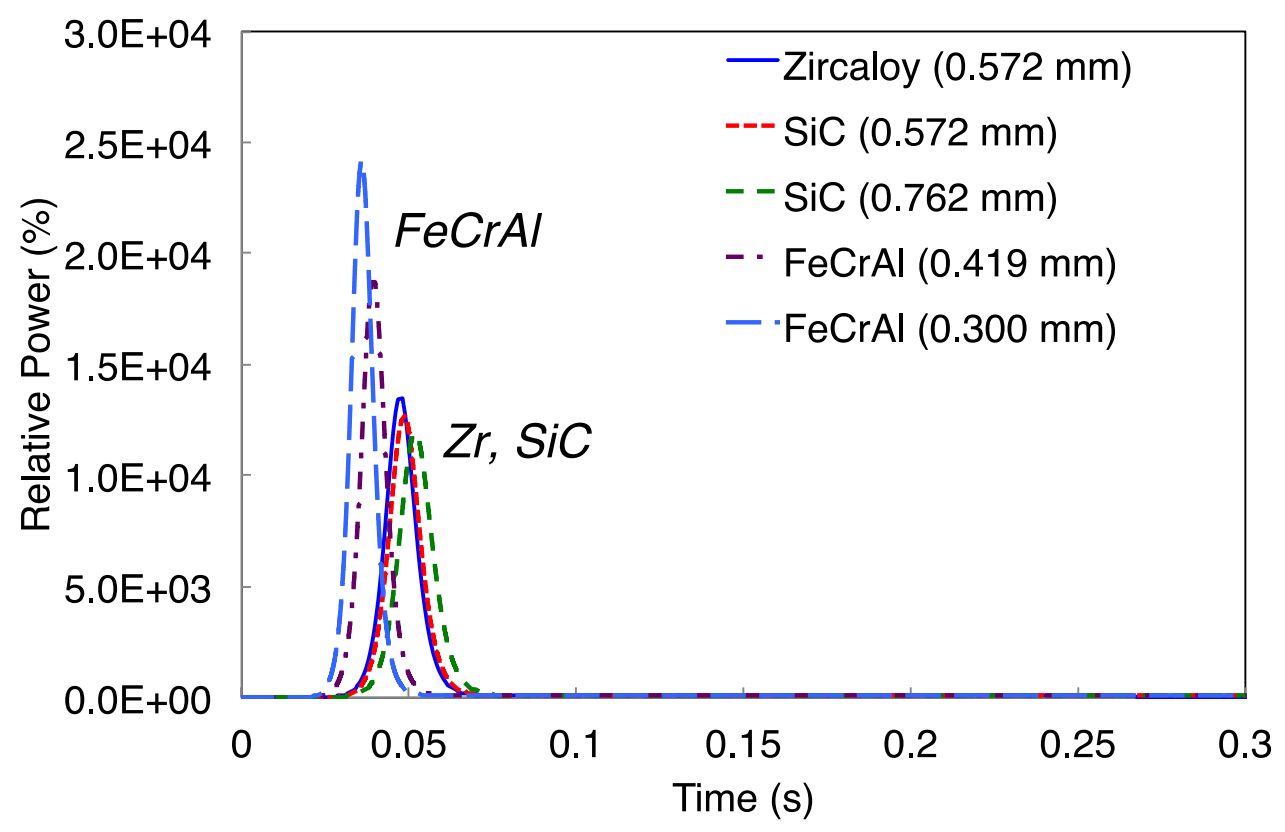

Figure 20. Super-prompt critical power response, Case 2. 


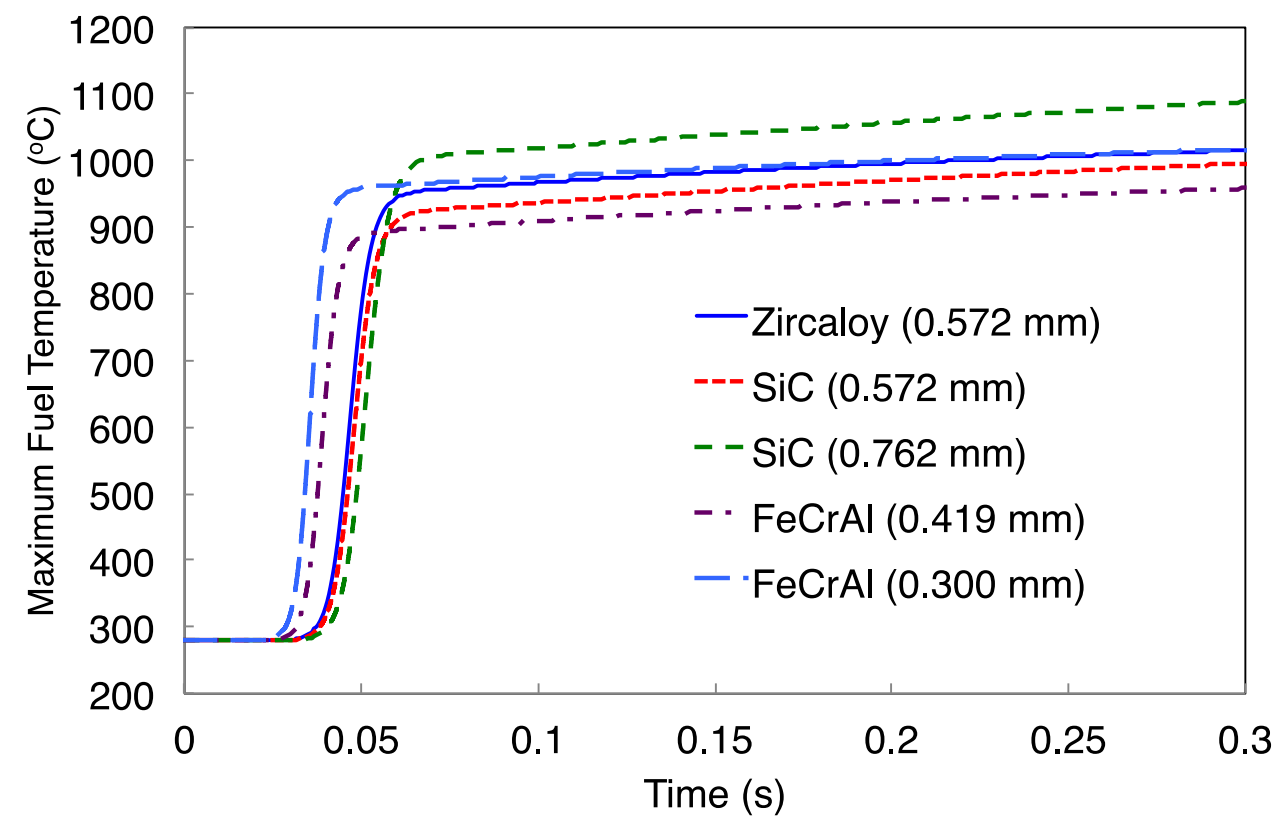

Figure 21. Super-prompt critical maximum fuel temperature increase, Case 2.

\subsection{Results for Case 3: Different Total Reactivity Worth, Delayed Ejection}

Case 3 shows an additional impact of the different rod worth in Case 2 by ejecting the control rods over an assumed period of 0.1 seconds with constant control rod motion over this period. This time delay was selected for the purpose of a sensitivity study, and it not necessarily a realistic ejection time. This results in different reactivity insertion rates, which correspond to additional differences in RIA response. An instantaneous ejection of control rods is not possible, and a physically reasonable hypothesized control rod ejection event would occur over some brief time period. The reactivity insertions are shown in Figure 22, and the power pulse response is shown in Figure 23. The corresponding increase in maximum fuel temperature (averaged across the assembly for the peak 
axial location in the core) is shown in Figure 24. The results are similar to Case 2, with a short time delay due to the 0.1 -second control rod ejection. The differences are more pronounced than in Case 1 due to the variation in control rod worth.

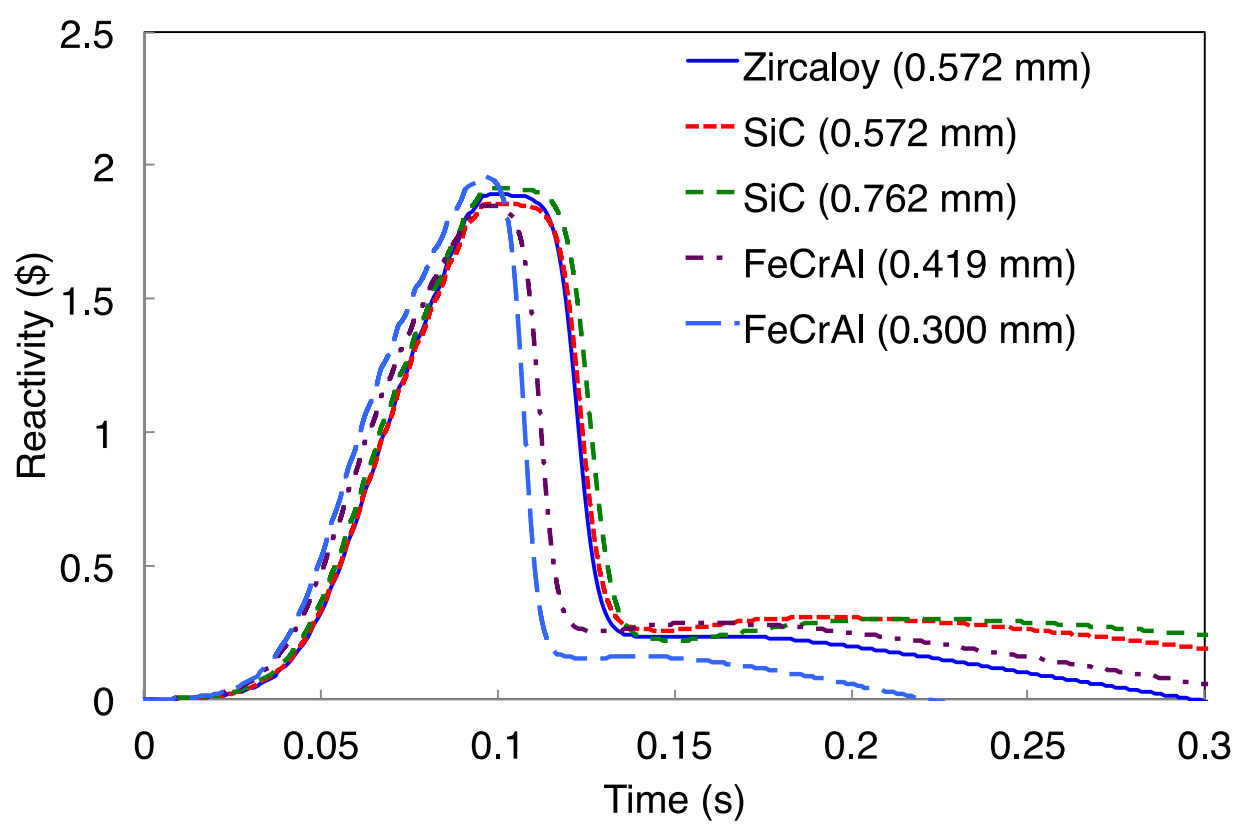

Figure 22. Super-prompt critical reactivity step insertion, Case 3. 


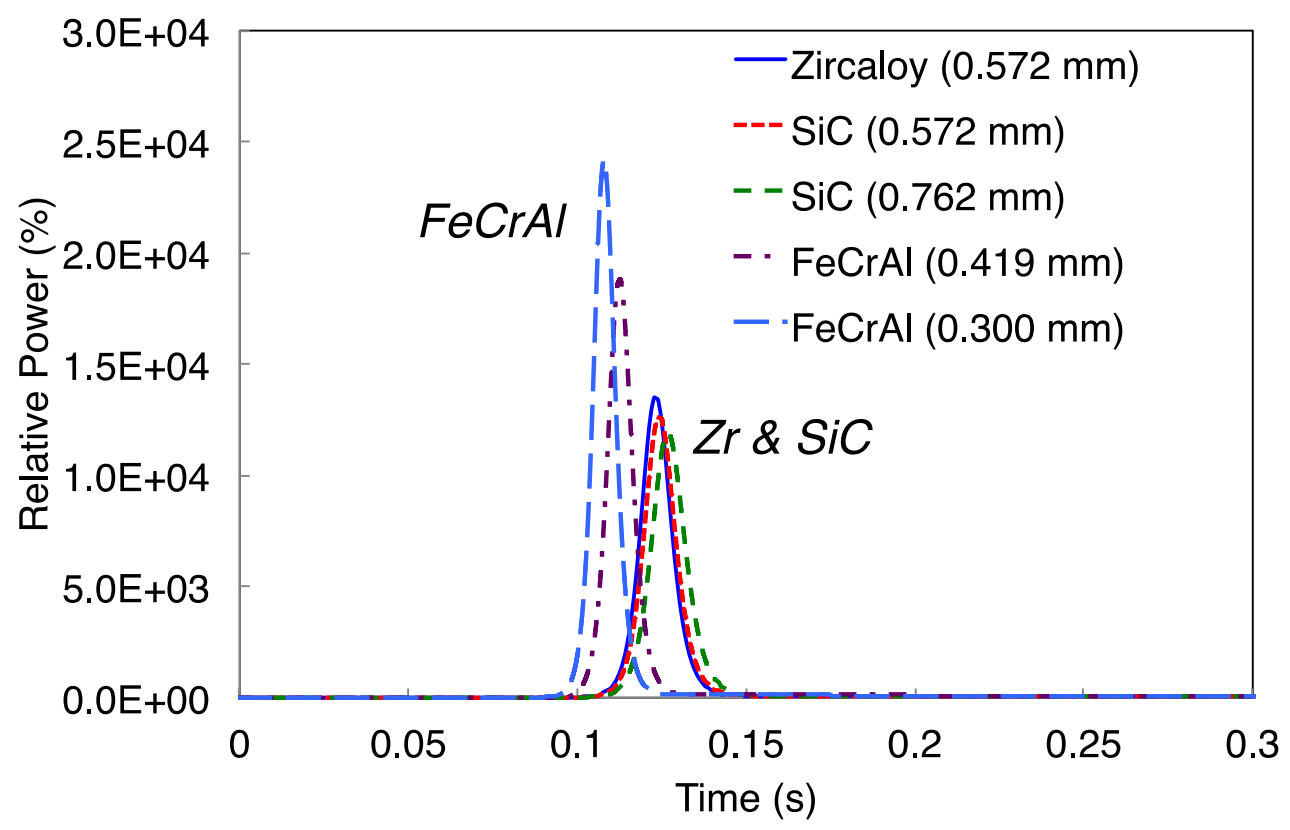

Figure 23. Super-prompt critical power response, Case 3. 


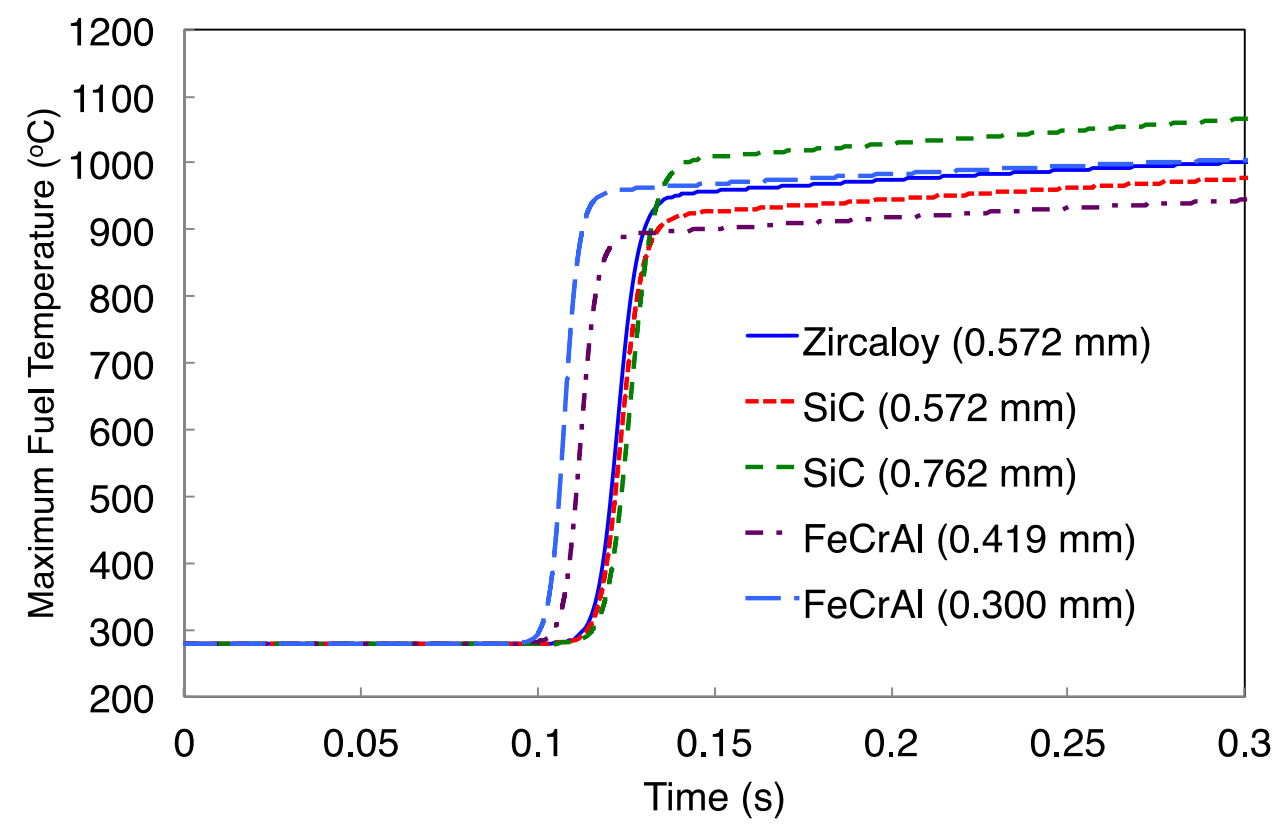

Figure 24. Super-prompt critical maximum fuel temperature increase, Case 3.

\section{Comparison with the Nordheim-Fuchs Analytical Model}

A Nordheim-Fuchs analytical model was compared with the results from the spatial kinetics calculations. The objective was to compare the detailed reactor kinetics simulations to a simplified model that can be used for scoping studies. The analytical model will be used in the following section to quantify, on a scoping basis, the differences of the reactor models in RIA response for each burnup point in the equilibrium cycle of the reactor models.

The Nordheim-Fuchs model employs several simplifying assumptions to obtain approximate analytical formulas for peak power, time of peak power, and full width at half maximum. The most significant approximations include the neglect of contributions from delayed neutrons, use of 
constant fuel volumetric heat capacity, use of constant fuel temperature coefficients, and assumption of a constant power shape throughout the RIA transient. By contrast, the PARCS spatial kinetics solution accounts for the change in power shape (due primarily to control-rod insertion), as well as temperature dependence of the fuel volumetric heat capacity and fuel temperature coefficients. As a result, a discrepancy between the Nordheim-Fuchs analytical model and spatial kinetics solutions is to be expected.

Table 8 shows the Nordheim-Fuchs analytical results for an instantaneous $\$ 1.74$ reactivity insertion. The point kinetics parameters given in Table 2 were used, with the exception of the fuel temperature coefficient, which was scaled by a constant multiplier for all cases to compensate for the discrepancy caused by the Nordheim-Fuchs simplifying point reactor assumptions. The multiplier was selected to account for differences between the Nordheim Fuchs model and the PARCS spatial kinetics solution and was applied uniformly to all cases. Once the multiplier was applied, the results showed consistent agreement with the spatial kinetics solution for all five cladding configurations, with a maximum relative error of $6.4 \%$ in any of the output parameters. This consistency in results gave confidence that the governing physics were effectively captured by the Nordheim-Fuchs model, allowing for its use as a general scoping tool for the parametric studies shown in the following section.

Table 8. Summary of Nordheim-Fuchs analytical results and relative differences versus 3-D kinetics.

\begin{tabular}{llllll}
\hline Cladding material & Zircaloy & SiC/SiC & SiC/SiC & FeCrAl & FeCrAl \\
\hline Cladding thickness $(\mathbf{m m})$ & 0.572 & 0.572 & $\mathbf{0 . 7 6 2}$ & $\mathbf{0 . 4 1 9}$ & $\mathbf{0 . 3 0 0}$ \\
Time of peak power $(\mathbf{s})$ & $5.36 \mathrm{E}-02$ & $5.31 \mathrm{E}-02$ & $5.99 \mathrm{E}-02$ & $4.27 \mathrm{E}-02$ & $4.34 \mathrm{E}-02$ \\
\hline
\end{tabular}




\begin{tabular}{|c|c|c|c|c|c|}
\hline Peak power ( $\%$ of nominal) & $1.00 \mathrm{E}+04$ & $1.01 \mathrm{E}+04$ & $8.39 \mathrm{E}+03$ & $1.39 \mathrm{E}+04$ & $1.39 \mathrm{E}+04$ \\
\hline Full width at half maximum (s) & $1.29 \mathrm{E}-02$ & $1.28 \mathrm{E}-02$ & $1.45 \mathrm{E}-02$ & $1.01 \mathrm{E}-02$ & $1.02 \mathrm{E}-02$ \\
\hline \multicolumn{6}{|c|}{ Differences relative to 3-D reactor kinetics simulation } \\
\hline Time of peak power $(s)$ & $-2.60 \%$ & $-2.80 \%$ & $-2.80 \%$ & $-2.80 \%$ & $-3.20 \%$ \\
\hline Peak power ( $\%$ of nominal) & $3.30 \%$ & $3.40 \%$ & $3.30 \%$ & $-5.70 \%$ & $-5.40 \%$ \\
\hline Full width at half maximum (s) & $5.20 \%$ & $5.00 \%$ & $4.60 \%$ & $6.30 \%$ & $6.40 \%$ \\
\hline
\end{tabular}

\section{Parametric Study Using the Nordheim-Fuchs Analytical Model}

Kinetics parameters as a function of time during the equilibrium cycle were extracted from the nodal core calculation at 10-day depletion intervals at HFP conditions. Table 9 summarizes the relative change in each kinetics parameter from the beginning to the end of the cycle. The impact of the changes in kinetics parameters on the RIA behavior are shown in Figure 25 through Figure 27. During the equilibrium cycle, the increase in fuel temperature coefficient, increase in neutron generation time, and decrease in delayed neutron fraction (while maintaining a constant $\$ 1.74$ reactivity insertion) all contributed to a decrease in peak power. The increase in neutron generation time and decrease in delayed neutron fraction also contributed to the increases in the time of peak power and full width at half maximum. Because the peak power decreases with burnup while the time of peak power increases, the fuel expansion rate for a super-prompt RIA is predicted to be highest at the beginning of the cycle and lowest at the end. The total unconstrained fuel thermal expansion is expected to decrease slightly throughout the cycle as well. Note that this analysis does not account for changes in gap size or other thermo-mechanical considerations. 
Table 9. Relative change in kinetics parameters and super prompt RIA results during the equilibrium cycle.

\begin{tabular}{|c|c|c|c|c|c|}
\hline Cladding material & Zircaloy & $\mathrm{SiC} / \mathrm{SiC}$ & $\mathrm{SiC} / \mathrm{SiC}$ & FeCrAl & FeCrAl \\
\hline Cladding thickness (mm) & 0.572 & 0.572 & 0.762 & 0.419 & 0.300 \\
\hline FTC & $8 \%$ & $8 \%$ & $10 \%$ & $9 \%$ & $9 \%$ \\
\hline$\Lambda$ & $20 \%$ & $21 \%$ & $23 \%$ & $16 \%$ & $14 \%$ \\
\hline $\boldsymbol{\beta}_{\text {eff }}$ & $-9 \%$ & $-9 \%$ & $-10 \%$ & $-9 \%$ & $-9 \%$ \\
\hline \multicolumn{6}{|c|}{ Relative change in super-prompt RIA results during the equilibrium cycle } \\
\hline Time of peak power & $20 \%$ & $20 \%$ & $22 \%$ & $17 \%$ & $16 \%$ \\
\hline Peak power & $-36 \%$ & $-36 \%$ & $-38 \%$ & $-33 \%$ & $-32 \%$ \\
\hline Full width at half maximum & $32 \%$ & $33 \%$ & $36 \%$ & $27 \%$ & $25 \%$ \\
\hline
\end{tabular}

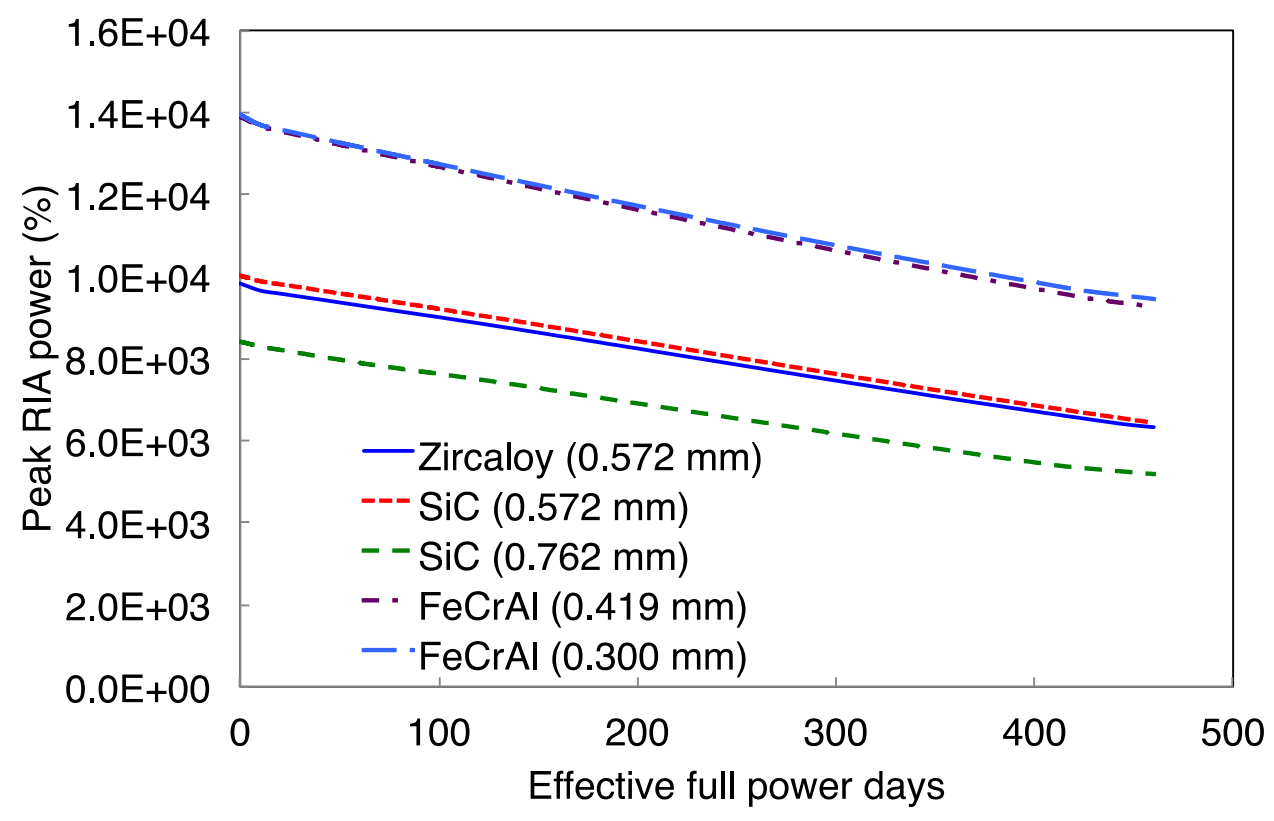

Figure 25. Peak power for a \$1.74 RIA event occurring at different points in the equilibrium cycle. 


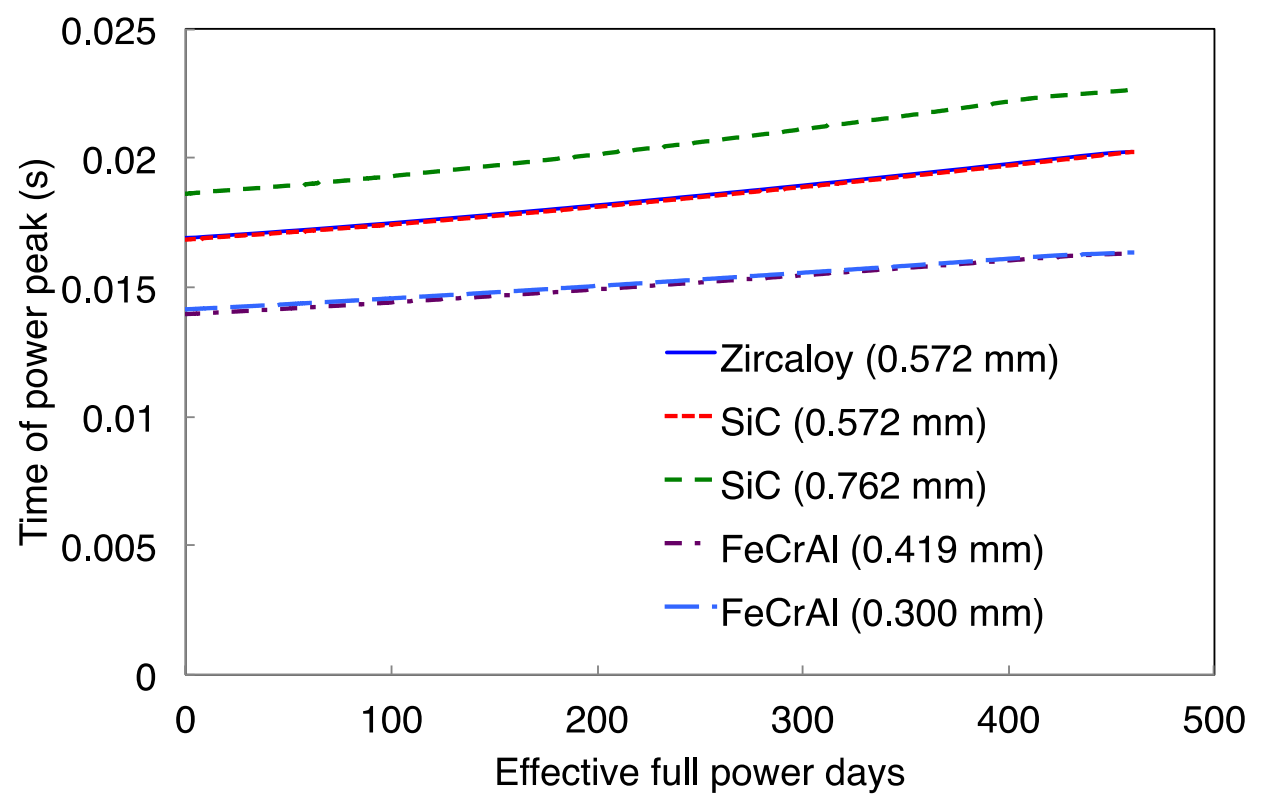

Figure 26. Time of peak power for a \$1.74 RIA event occurring at different points in the equilibrium cycle. 


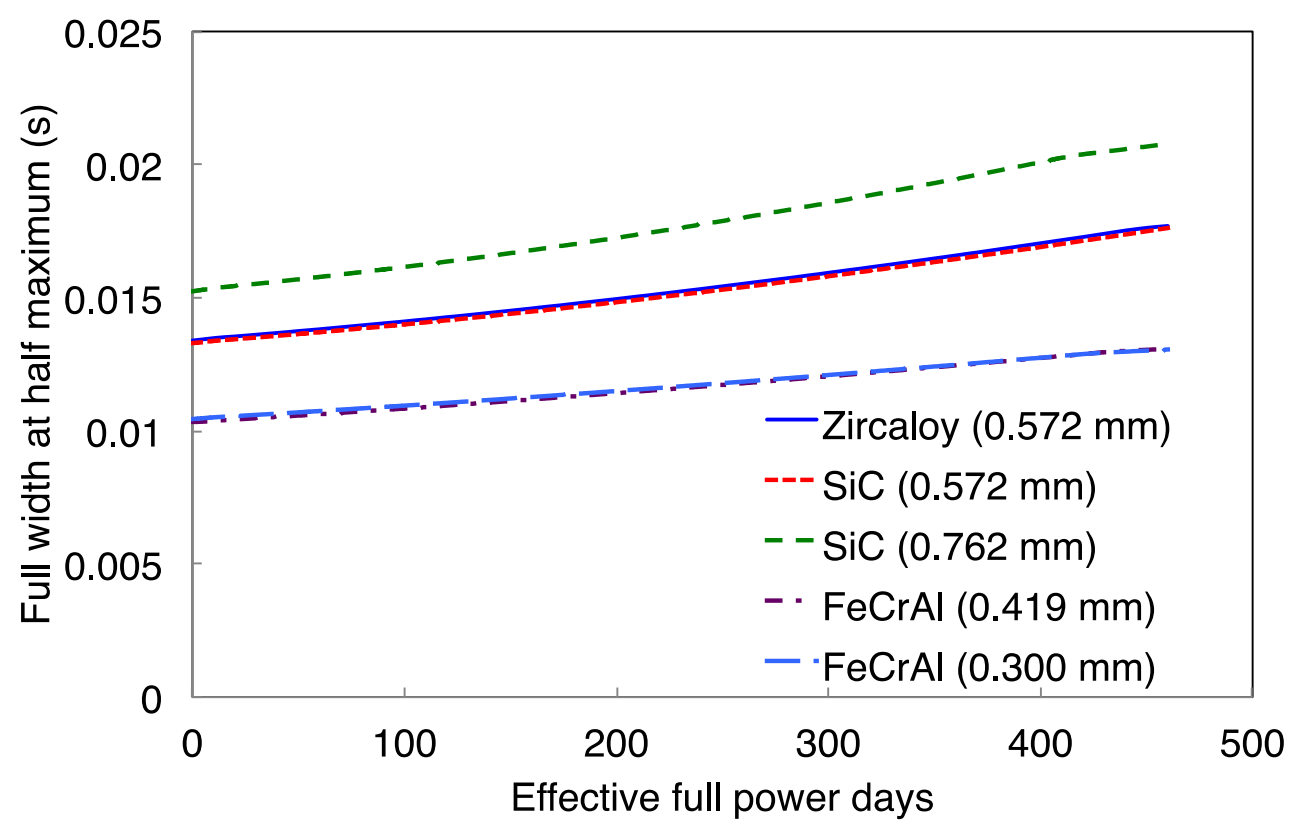

Figure 27. Full width at half maximum for a \$1.74 RIA event occurring at different points in the equilibrium cycle.

\section{Future Work}

Additional efforts are necessary to support transient testing of these and other ATF concepts.

PCMI experiments are needed to evaluate the response of fresh and irradiated cladding materials as a function of strain and strain rate. Consideration and measurement of thermal hydraulic characteristics, including critical heat flux, is important to understand the high temperature phase of the RIA transient. Translating fuel strain into cladding strain requires modeling of the integral fuel response. Modeling of the integral fuel response to these events is another key aspect of future work. This modeling will use the Nuclear Energy Advanced Modeling and Simulation (NEAMS) tool, BISON. Each of these 
future activities are conducted in support of eventual integral irradiation experiments in the TREAT reactor at Idaho National Laboratory.

In support of the AFC ATF program, a PCMI separate effects test device is being developed and tested at Oak Ridge National Laboratory (ORNL) to provide information on potential PCMI-induced failure of candidate cladding materials. The device is based on an improved version of a modified burst test facility design presented in Yueh et al. (2012) and Yueh et al. (2016). The various methods of cladding burst testing are thoroughly reviewed in Desquines et al. (2012). The objective of the test device is to provide information on the PCMI phase of the RIA transient using a driver tube to simulate a rapidly expanding fuel pellet. The AFC PCMI separate-effects test device is shown in Figure 28. This modified burst test device uses a pressurized driver tube that simulates PCMI by expanding and producing a resultant strain in the cladding. Details on the operation and design of the device are outside the scope of the present paper but will be presented in future publications. The applicability of tests results obtained from this device depends on the degree to which the device simulates prototypic stresses and conditions on test samples and the degree to which the conditions being simulated are appropriate for the events being considered. The PCMI separate effects test device is an example of a test capability that will benefit from RIA conditions calculated in this study. 


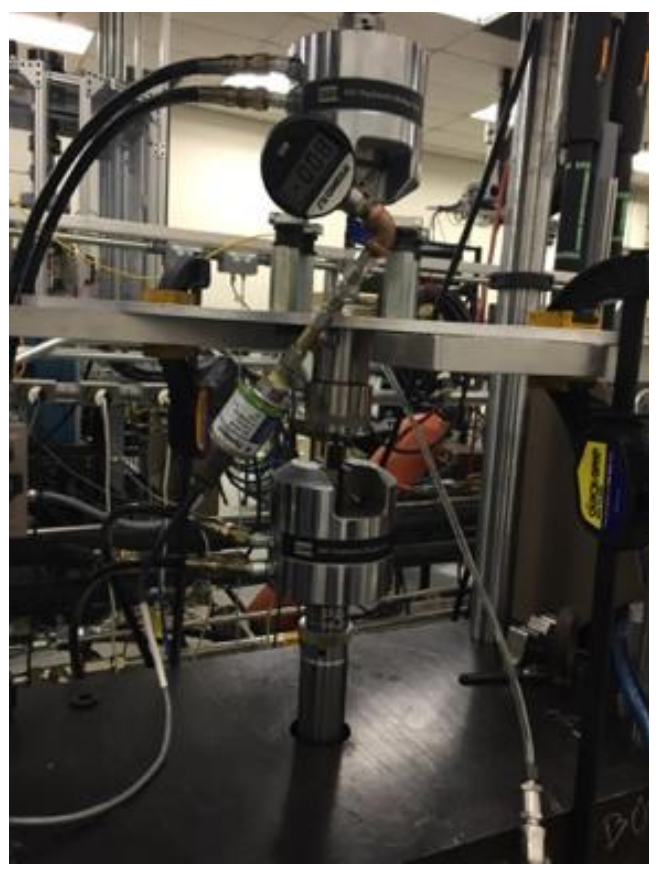

Figure 28. AFC modified burst test device.

\section{Summary}

To inform planned tests of PCMI in RIA for potential advanced cladding materials, three-dimensional nodal core models were developed to account for thermal and hydraulic feedback. The core models are in a three-batch equilibrium cycle with approximately $5 \%$ enrichment for all but one case with $\mathrm{FeCrAl}$ cladding. This modeling is necessary for informed transient test design and is an important part of nuclear and nonnuclear transient testing such as that to be performed at ORNL using a PCMI separate effects test device.

Cladding material and fuel design make a difference for RIA response, including core response and fuel rod response. This study identified distinct differences in the behavior of the cases with 
FeCrAl cladding from the Zircaloy and $\mathrm{SiC} / \mathrm{SiC}$ cases. This paper demonstrates an approach to determine the parameters needed to simulate RIA test conditions. These simulations help to draw out distinctions between responses of different fuel and cladding designs.

The RIA simulations indicate that the predicted RIA energy deposition is similar for the Zircaloy, $\mathrm{FeCrAl}$, and $\mathrm{SiC} / \mathrm{SiC}$ cladding options. The total thermal expansion of the fuel is similar because it is a function of the energy deposition. However, the predicted maximum fuel diameter change rate is higher and the pulse is greater in magnitude with reduced full width at half maximum for $\mathrm{FeCrAl}$ cladding. These differences result from the greater fuel-to-moderator ratio in the FeCrAl configurations considered in this paper, as well parasitic absorption in iron and chromium. This hardened spectrum drives a shorter neutron generation time, yielding impacts on pulse width and timing, which will impact the strain rate that the cladding would experience in a PCMI event.

\section{Acknowledgements}

This effort was supported by the US DOE-NE Advanced Fuels Campaign. M. N. Cinbiz, T. J. Harrison, K. R. Robb, and D. C. Crawford of ORNL are gratefully acknowledged for their internal review of this paper and the work.

\section{References}

Alvis, J., Liu, W. and Montgomery, R. "Fuel Reliability Program: Proposed RIA Acceptance Criteria, Report No. 1021036." Electric Power Research Institute, Palo Alto, CA (2010).

Andrews, N., Shirvan, K., Pilat, E. E., and Kazimi, M. S., "Steady State and Accident Transient 
Analysis Burning Weapons-Grade Plutonium in Thorium and Uranium with Silicon Carbide Cladding." Nuclear Technology, 1942 (2016): 204-216.

Brown, N.R., Ludewig, H., Aronson, A., Raitses, G. and Todosow, M., 2013. Neutronic evaluation of a PWR with fully ceramic microencapsulated fuel. Part I: Lattice benchmarking, cycle length, and reactivity coefficients. Annals of Nuclear Energy, 62 (2013), 538-547.

Brown, N. R., Aronson, A., Todosow, M., Brito, R., and McClellan, K. J., "Neutronic Performance of Uranium Nitride Composite Fuels in a PWR," Nuclear Engineering and Design, 275 (2014): 393-407.

Brown, N. R., Todosow, M., and Cuadra, A., "Screening of Advanced Cladding Materials and UN-U 3 $\mathrm{Si}_{5}$ Fuel," Journal of Nuclear Materials 462 (2015): 26-42.

Carpenter, D. M., An assessment of silicon carbide as a cladding material for light water reactors (Doctoral dissertation, Massachusetts Institute of Technology) (2010).

DeHart, M. D., and Bowman, S. M.. "Reactor Physics Methods and Analysis Capabilities in SCALE," Nuclear Technology 174 (2011): 196-213.

Desquines, J., Koss, D. A., Motta, A. T., Cazalis, B., and Petit, M, “The Issue of Stress State during Mechanical Tests to Assess Cladding Performance during a Reactivity-Initiated Accident (RIA)," Journal of Nuclear Materials 412, 2 (2011): 250-267.

Dobisesky, J. P., Reactor physics considerations for implementing silicon carbide cladding into a PWR environment (Doctoral dissertation, Massachusetts Institute of Technology) (2011). 
Downar, T. J., Barber, D. A., Miller, R. M., et al.. PARCS: Purdue advanced reactor core simulator, In: Proceedings of the International Meeting on New Frontiers of Nuclear Technology: Reactor Physics, Safety and High-Performance Computing, PHYSOR 2002, Seoul.

Electric Power Research Institute. Topical Report on Reactivity Initiated Accident: Bases for RIA Fuel and Core Coolability Criteria, EPRI, Palo Alto (2002).

Fuketa, T. "Transient Response of LWR Fuels (RIA)," Comprehensive Nuclear Materials, Konings, R., Ed. Elsevier: 2011.

George, N. M., Terrani, K., Powers, J., Worrall, A., and Maldonado, I, "Neutronic Analysis of Candidate Accident-Tolerant Cladding Concepts in Pressurized Water Reactors," Annals of Nuclear Energy 75 (2015): 703-712.

Goldner, F. Development strategy for advanced LWR fuels with enhanced accident tolerance. In Enhanced Accident Tolerant LWR Fuels National Metrics Workshop, Germantown, MD (2012).

Hursin, M., Downar, T. J., and Montgomery, R, "Impact of improved neutronic methodology on the cladding response during a PWR reactivity initiated accident." Nuclear Engineering and Design, 262 (2013): 180-188.

Jessee, M.A., Wieselquist, W.A., Evans, T.M., Hamilton, S.P., Jarrell, J.J., Kim, K.S., Lefebvre, J.P., Lefebvre, R.A., Mertyurek, U., Thompson, A.B. and Williams, M.L, "Polaris: A New Two-Dimensional Lattice Physics Analysis Capability for the SCALE Code System.” Proc. PHYSOR 2014 (2014). 
Katoh, Y., Ozawa, K., Shih, C., Nozawa, T., Shinavski, R. J., Hasegawa, A., Snead, L. L. “Continuous SiC fiber, CVI SiC matrix composites for nuclear applications: Properties and irradiation effects.” Journal of Nuclear Materials 448, no. 1 (2014): 448-476.

Kozlowski, T., and Downar, T. J., PWR MOX/UO ${ }_{2}$ Core Transient Benchmark Final Report. Purdue University, ISBN 92-64-02330-5 NEA/NSC/DOC 20 (2006).

Lee, Y., McKrell, T. J., Yue, C., and Kazimi, M. S., "Safety assessment of SiC cladding oxidation under loss-of-coolant accident conditions in light water reactors." Nuclear Technology, 183 2, (2013): $210-227$.

Luscher, W. G., Beyer, C. E., and Cuta, J. M., "FRAPTRAN 1.4: A Computer Code for the Transient Analysis of Oxide Fuel Rods," NUREG/CR-7023, vol. 1; PNNL-19400, vol. 1, US Nuclear Regulatory Commission, Office of Nuclear Regulatory Research (2011).

Sonnenburg, H.G., Analyses of $\mathrm{UO}_{2}$ RIA-Tests with the Code TESPA-ROD, GRS-A-8574, Gesellschaft für Anlagen-und Reaktorsicherheit, Germany, Garching (2008).

Sugiyama, T., Umeda, M., Fuketa, T., Sasajima, H., Udagawa, Y., Nagase, F. "Failure of high burnup fuels under reactivity-initiated accident conditions," Annals of Nuclear Energy 36, no. 3 (2009): $380-385$.

Terrani, K. A., Zinkle, S. J., and Snead, L. L., “Advanced oxidation-resistant iron-based alloys for LWR fuel cladding," Journal of Nuclear Materials, 4481 (2014): 420-435.

Todosow, M., et al., 2015. Report on Fuel Cycle Impacts of Accident Tolerant Fuel, 
FCRD-FCO-2015-000386.

U.S. Atomic Energy Commission. Regulatory Guide 1.77-Assumptions used for evaluating a control rod ejection accident for pressurized water reactors. (1974).

U. S. Nuclear Regulatory Commission. Standard Review Plan for the Review of Safety Analysis Reports for Nuclear Power Plants, LWR Edition, NUREG-0800, Section 4.2 Fuel System Design, Rev. 3, ; U. S. Nuclear Regulatory Commission, Office of Nuclear Reactor Regulation: March 2007.

Yamamoto, Y., et al. "Development and property evaluation of nuclear grade wrought $\mathrm{FeCrAl}$ fuel cladding for light water reactors." Journal of Nuclear Materials 467 (2015): 703-716.

Younker, I., Fratoni, M. "Neutronic evaluation of coating and cladding materials for accident tolerant fuels." Progress in Nuclear Energy 88 (2016): 10-18.

Yueh, H. K., Karlsson, J., Lees, W., Mitchell, D., and Quecedo, M., "New Techniques for the Testing of Cladding Material Under RIA Conditions," in Proceedings of Top Fuel 2012, Manchester, UK, September 2-6.

Yueh, K., et al. "Fuel cladding behavior under rapid loading conditions." Journal of Nuclear Materials 469 (2016): 177-186.

Zinkle, S. J., Terrani, K. A., Gehin, J. C., Ott, L. J., Snead, L. L., “Accident Tolerant Fuels for LWRs: A Perspective," Journal of Nuclear Materials, 448, 1 (2014): 374-379. 